\title{
INFERENCES BASED ON THE GROUPED OBSERVATIONS FROM THE BIVARIATE POWER-NORMAL DISTRIBUTION
}

\author{
Toshimitsu Hamasaki* and Masashi Goto ${ }^{\dagger}$
}

\begin{abstract}
In this paper, we have investigated the relationship between the two variables in which observations of the two variables were grouped into several intervals, when the bivariate power-normal distribution proposed by Goto et al. (1980) were fitted to these observations. The results of some numerical examples showed that the bivariate powernormal distribution would be helpful to "regularize" the observations even when the strict bivariate normality was not achieved. Also, a bivariate normal problem was considered in the framework developed in fitting the bivariate power-normal distribution to the grouped observations.
\end{abstract}

\section{Introduction}

In medical science and biology, observations may be specified only by two observed points (each observation being defined by upper and lower bounds) because of some restrictions during the process of phenomenon occurring, and observation systems. Also, observations are grouped into the intervals in advance by some criteria. For example, observations may be of some property connected with the health of the patient in clinical trials, so that patients are classified as "absent", "mild", "moderate", and "serve" (Cox, 1957). Furthermore, it is desirable for ease of calculation or graphical representation to divide the observed range of variation into classes (Cox, 1957: Borkowf, et al., 1997). In this case, statistical processings are often carried out on the grouped observations. For example, the midpoint in the interval is handled as a representative value, and some corrections such as Sheppard may be used to calculate some statistics or quantities such as mean and variance (Stuart and Ord, 1986). However, this procedure may not often be appropriate in practice because skewed distribution is often observed, the numbers of intervals are often small, and the length of intervals are not uniform.

So, the probability that the observations lie in the interval is obtained by supposing some underlying distributions being appropriate for describing the phenomenon. Thus, the likelihood is constructed and the inferences about parameters of distribution are derived on the likelihood. In this case, the inferences about the parameters which describe the shape of distribution are focus (Kulldorff, 1962: Kempthorne, 1966: Barnard, 1976: Kempthorne and Folks, 1971: Copas, 1972: Giesbrecht and Kempthorne, 1976: Nabeya, 1983: Stuart and Ord, 1986: Atkinson et al., 1991). Then, goodness of fit test on chi-square statistics,

* Clinical Statistics Group, Biometrics, Central Research Clinical Development Japan, Pfizer Pharmaceuticals Inc., P. O. Box 226, Mitsui Bldg., 2-1-1 Nishi-Shinjuku, Shinjuku-ku, Tokyo, 163-0461, Japan.

$\dagger$ Department of Informatics and Mathematical Science, Graduate School of Engineering Sciences, Osaka University, 1-3 Machikaneyama-cho, Toyonaka, Osaka 560-8531, Japan.

Key words: bivariate normal regression: correlation table; power-transformation; simple regression 
or information criteria such as AIC are used to select the underlying distribution (Kariya, 1975, 1979: Kariya and Akasaka, 1976: Sugiura, 1980, 1981: Domae and Miyahara, 1984). Since such measures are used under these underlying distributions, the gap between the real and the ideal hypothesis can be quantitatively represented and evaluated. However, it is hardly flexible to try fitting of the distributions on data-adaptive so far as one persist in these underlying distributions. In practice, it is desirable that the inference can be carried out on the family of the distribution or the data-adaptive distribution which includes the underlying distribution, if possible, the normal distribution with historical and statistical "property" (Matsubara and Goto, 1979: Goto, 1986).

In this paper, the investigations on the fitting power-normal distribution (PND) to the grouped observations (Hamasaki and Goto, 1997, 1998) are extended to a bivariate case. Namely the bivariate power-normal distribution (BPND), an extension of the power-normal distribution to two-dimensional case is applied to the bivariate grouped observations. This is due to three reasons, i.e., (i) a bivariate case will be the base of the extension to a multivariate case, (ii) the structure of a bivariate case can be relatively and easily comprehensible, and (iii) a difference in the structure between a univariate and a bivariate case can be clearly shown.

In section 2, the definition and properties of the BPND are briefly mentioned. And the procedure of fitting the BPND to the grouped observation is developed. In section 3 , the performances are investigated and illustrated with some numerical example cited form the published literatures. In section 4 , a bivariate normal regression problem is considered in the framework developed in section 2. Also, a simple regression problem is mentioned. In section 5 , the some knowledge obtained in this investigation are summarized as concluding remarks.

\section{Fitting of the BPND to the Grouped Observations}

\subsection{The Bivariate Power-normal Distribution}

A relationship between two positive random variables $\left(Y_{1}, Y_{2}\right)$ is considered, where $0<Y_{1}, Y_{2}<\infty$. Then, the power-transformed variables $\left(Y_{1}^{\left(\lambda_{1}\right)}, Y_{2}^{\left(\lambda_{2}\right)}\right)$ of $\left(Y_{1}, Y_{2}\right)$ have nearly a bivariate normal. The reason why the power-transformed variables do not have a strict bivariate normal is because $Y_{1}^{\left(\lambda_{1}\right)}$ and $Y_{2}^{\left(\lambda_{2}\right)}$ are bounded or truncated according to the transforming parameters $\boldsymbol{\lambda}=\left(\lambda_{1}, \lambda_{2}\right)^{\mathrm{T}}$. For example, $Y_{1}>0$ implies $Y_{1}^{\left(\lambda_{1}\right)}<-1 / \lambda_{1}$ if $\lambda_{1}>0$ or $Y_{1}^{\left(\lambda_{1}\right)}<-1 / \lambda_{1}$ if $\lambda_{1}<0$, thus, for $\lambda_{1} \neq 0, Y_{1}^{\left(\lambda_{1}\right)}$ is bounded and hence strictly speaking, $Y_{1}^{\left(\lambda_{1}\right)}$ can not have a normal distribution. Allowed for such a truncation, $\left(Y_{1}, Y_{2}\right)$ has the bivariate power-normal distribution (BPND), which is the extension of power-normal distribution (PND) proposed by Goto et al. (1983) to a bivariate case, whose probability density function is given by

$$
g\left(y_{1}, y_{2}\right)=\frac{y_{1}^{\lambda_{1}-1} y_{2}^{\lambda_{2}-1}}{\Lambda(\boldsymbol{\lambda}, \boldsymbol{\mu}, \boldsymbol{\Sigma})} f\left(y_{1}^{\left(\lambda_{1}\right)}, y_{2}^{\left(\lambda_{2}\right)}\right), \quad 0<y_{1}, y_{2}<\infty
$$

where

$$
f\left(y_{1}^{\left(\lambda_{1}\right)}, y_{2}^{\left(\lambda_{2}\right)}\right)=\frac{1}{2 \pi \sigma_{1} \sigma_{2} \sqrt{1-\rho^{2}}} \exp \left\{-\frac{1}{2} Q\left(y_{1}^{\left(\lambda_{1}\right)}, y_{2}^{\left(\lambda_{2}\right)}\right)\right\}
$$


Inferences Based on the Grouped Observations from The Bivariate Power-Normal Distribution

$$
\begin{aligned}
& Q\left(y_{1}^{\left(\lambda_{1}\right)}, y_{2}^{\left(\lambda_{2}\right)}\right)=\frac{1}{1-\rho^{2}} \\
& \quad \times\left\{\left(\frac{y_{1}^{\left(\lambda_{1}\right)}-\mu_{1}}{\sigma_{1}}\right)^{2}-2 \rho\left(\frac{y_{1}^{\left(\lambda_{1}\right)}-\mu_{1}}{\sigma_{1}}\right)\left(\frac{y_{2}^{\left(\lambda_{2}\right)}-\mu_{2}}{\sigma_{2}}\right)+\left(\frac{y_{2}^{\left(\lambda_{2}\right)}-\mu_{2}}{\sigma_{2}}\right)^{2}\right\} \\
& y_{1}^{\left(\lambda_{1}\right)}=\left\{\begin{array}{cc}
\frac{y_{1}^{\lambda_{1}}-1}{\lambda_{1}}, & \lambda_{1} \neq 0, \\
\log y_{1}, & \lambda_{1}=0
\end{array} \text { and } y_{2}^{\left(\lambda_{2}\right)}=\left\{\begin{array}{cc}
\frac{y_{2}^{\lambda_{2}}-1}{\lambda_{2}}, & \lambda_{2} \neq 0 \\
\log y_{2}, & \lambda_{2}=0
\end{array}\right.\right.
\end{aligned}
$$

(Goto et al., 1980), where $\boldsymbol{\mu}$ is the vector of mean parameter and $\boldsymbol{\Sigma}$ is the variance-covariance matrix in which $\left(Y_{1}^{\left(\lambda_{1}\right)}, Y_{2}^{\left(\lambda_{2}\right)}\right)$ has nearly a bivariate normal distribution and are given by

$$
\boldsymbol{\mu}=\left(\mu_{1}, \mu_{2}\right)^{\mathrm{T}}
$$

and

$$
\boldsymbol{\Sigma}=\left(\begin{array}{cc}
\sigma_{1}^{2} & \rho \sigma_{1} \sigma_{2} \\
\rho \sigma_{1} \sigma_{2} & \sigma_{1}^{2}
\end{array}\right)
$$

respectively. $A(\boldsymbol{\lambda}, \boldsymbol{\mu}, \boldsymbol{\Sigma})$ is the probability proportional to constant term of the BPND and is presented by

$$
A(\boldsymbol{\lambda}, \boldsymbol{\mu}, \boldsymbol{\Sigma})=\int_{a_{2}}^{b_{2}} \int_{a_{1}}^{b_{1}} \phi_{2}\left(u_{1}, u_{2}: \rho\right) d u_{1} d u_{2}
$$

in term of joint probability density function of the bivariate standard normal distribution ${ }^{1}$

$$
\phi_{2}\left(u_{1}, u_{2}: \rho\right)=\frac{1}{2 \pi \sqrt{1-\rho^{2}}} \exp \left\{-\frac{u_{1}^{2}-2 \rho u_{1} u_{2}+u_{2}^{2}}{2\left(1-\rho^{2}\right)}\right\} .
$$

Table 2.1.1. The values of $a_{1}, a_{2}, b_{1}$, and $b_{2}$

\begin{tabular}{c|c|cc|cc}
\hline$\lambda 1$ & $\lambda 2$ & $a_{1}$ & $b_{1}$ & $a_{2}$ & $b_{2}$ \\
\hline \multirow{4}{*}{$\lambda_{1}<0$} & $\lambda_{2}<0$ & & & $-\infty$ & $-\kappa_{2}$ \\
& $\lambda_{2}=0$ & $-\infty$ & $-\kappa_{1}$ & $-\infty$ & $\infty$ \\
& $\lambda_{2}>0$ & & & $-\infty$ & $\infty$ \\
\hline \multirow{3}{*}{$\lambda_{1}=0$} & $\lambda_{2}<0$ & & & $-\infty$ & $-\kappa_{2}$ \\
& $\lambda_{2}=0$ & $-\infty$ & $\infty$ & $-\infty$ & $\infty$ \\
& $\lambda_{2}>0$ & & & $-\infty$ & $\infty$ \\
\hline \multirow{3}{*}{$\lambda_{1}>0$} & $\lambda_{2}<0$ & & & $-\infty$ & $-\kappa_{2}$ \\
& $\lambda_{2}=0$ & $-\kappa_{1}$ & $\infty$ & $-\infty$ & $\infty$ \\
& $\lambda_{2}>0$ & & & $-\kappa_{2}$ & $\infty$ \\
\hline
\end{tabular}

Then, if the truncation points of $Y_{1}$ and $Y_{2}$ are $\kappa_{1}=\left(\lambda_{1} \mu_{1}+1\right) / \lambda_{1} \sigma_{1}$ and $\kappa_{2}=$ $\left(\lambda_{2} \mu_{2}+1\right) / \lambda_{2} \sigma_{2}$ respectively, $a_{1}, a_{2}, b_{1}$ and $b_{2}$ are given as Table 2.1.1. An actual magnitude

${ }^{1}$ Although an ordinary bivariate standard normal distribution is denoted by $\mathrm{N}_{2}(0, \mathrm{I})$, in this paper it is denoted by distribution $\mathrm{N}_{2}\left(0, \mathrm{I}^{*}\right)$ with variance-covariance matrix $\mathrm{I}^{*}=\left(\begin{array}{ll}1 & \rho \\ \rho & 1\end{array}\right)$, where 0 is the vector with its elements all equal to zero, I is the identity matrix. 


\section{HAMASAKI and GOTO}

of value of $A(\boldsymbol{\lambda}, \boldsymbol{\mu}, \boldsymbol{\Sigma})$ can be evaluated in term of the distribution function $\Phi_{2}(\cdot, \cdot: \rho)$ of a bivariate standard normal distribution and the distribution function $\Phi(\cdot)$ of a univariate standard normal distribution. For $\lambda_{1}<0, A(\boldsymbol{\lambda}, \boldsymbol{\mu}, \boldsymbol{\Sigma})$ is denoted by

$$
A(\boldsymbol{\lambda}, \boldsymbol{\mu}, \boldsymbol{\Sigma})= \begin{cases}\Phi_{2}\left(-\kappa_{1},-\kappa_{2}: \rho\right), & \lambda_{2}<0 \\ \Phi\left(-\kappa_{1}\right), & \lambda_{2}=0 \\ \Phi\left(-\kappa_{1}\right)-\Phi_{2}\left(-\kappa_{1},-\kappa_{2}: \rho\right), & \lambda_{2}>0\end{cases}
$$

for $\lambda_{1}=0$

$$
A(\boldsymbol{\lambda}, \boldsymbol{\mu}, \boldsymbol{\Sigma})= \begin{cases}1-\Phi\left(\kappa_{2}\right), & \lambda_{2}<0 \\ 1, & \lambda_{2}=0 \\ \Phi\left(\kappa_{2}\right), & \lambda_{2}>0\end{cases}
$$

and for $\lambda_{1}>0$

$$
A(\boldsymbol{\lambda}, \boldsymbol{\mu}, \boldsymbol{\Sigma})= \begin{cases}\Phi\left(\kappa_{1}\right)-\Phi_{2}\left(\kappa_{1}, \kappa_{2}: \rho\right), & \lambda_{2}<0 \\ \Phi\left(\kappa_{1}\right), & \lambda_{2}=0 \\ \Phi_{2}\left(\kappa_{1}, \kappa_{2}: \rho\right), & \lambda_{2}>0 .\end{cases}
$$

See Goto et al. (1980, 1981a, 1981b, 1982), Kawai et al. (1996), Jimura et al. (1996) for detailed discussions about the properties of the BPND.

Therefore, setting $\boldsymbol{\theta}^{\mathrm{T}}=\left(\lambda_{1}, \lambda_{2}, \mu_{1}, \mu_{2}, \sigma_{1}, \sigma_{2}, \rho\right)$, the log-likelihood function for the sample of size $n$ is given by

$$
\begin{aligned}
& \log L_{n}(\boldsymbol{\theta})=-n \log 2 \pi-\frac{n}{2}\left\{\log \sigma_{1}^{2}+\log \sigma_{2}^{2}+\log \left(1-\rho^{2}\right)\right\} \\
& -\frac{1}{2\left(1-\rho^{2}\right)} \sum_{i=1}^{n}\left\{\left(\frac{y_{1 l}^{\left(\lambda_{1}\right)}-\mu_{1}}{\sigma_{1}}\right)^{2}-2 \rho\left(\frac{y_{1 l}^{\left(\lambda_{1}\right)}-\mu_{1}}{\sigma_{1}}\right)\left(\frac{y_{2 l}^{\left(\lambda_{2}\right)}-\mu_{2}}{\sigma_{2}}\right)+\left(\frac{y_{2 l}^{\left(\lambda_{2}\right)}-\mu_{2}}{\sigma_{2}}\right)^{2}\right\} \\
& \quad+\left(\lambda_{1}-1\right) \sum_{i=1}^{n} \log y_{1 l}+\left(\lambda_{2}-1\right) \sum_{i=1}^{n} \log y_{2 l}-n \log A(\boldsymbol{\lambda}, \boldsymbol{\mu}, \boldsymbol{\Sigma}) .
\end{aligned}
$$

The maximum likelihood estimates of $\lambda_{1}, \lambda_{2}, \mu_{1}, \mu_{2}, \sigma_{1}, \sigma_{2}$, and $\rho$ are obtained by maximizing this log-likelihood function (2.1.12) over each of these parameters. Thus, assuming that $A(\boldsymbol{\lambda}, \boldsymbol{\mu}, \boldsymbol{\Sigma})=1$ for fixed $\lambda_{1}$ and $\lambda_{2}$, the maximum likelihood estimates of $\mu_{1}, \mu_{2}, \sigma_{1}^{2}$, $\sigma_{2}^{2}$, and $\rho$ are given by

$$
\begin{gathered}
\hat{\mu}_{1 n}\left(\lambda_{1}\right) \sum_{l=1}^{n} \frac{y_{1 l}^{\left(\lambda_{1}\right)}}{n}, \quad \hat{\mu}_{2 n}\left(\lambda_{2}\right) \sum_{l=1}^{n} \frac{y_{2 l}^{\left(\lambda_{2}\right)}}{n} \\
\hat{\sigma}_{1 n}^{2}\left(\lambda_{1}\right)=\sum_{l=1}^{n} \frac{\left\{y_{1 l}^{\left(\lambda_{1}\right)}-\hat{\mu}_{1 n}\left(\lambda_{1}\right)\right\}^{2}}{n}, \quad \hat{\sigma}_{2 n}^{2}\left(\lambda_{1}\right)=\sum_{l=1}^{n} \frac{\left\{y_{2 l}^{\left(\lambda_{2}\right)}-\hat{\mu}_{2 n}\left(\lambda_{2}\right)\right\}^{2}}{n}, \\
\hat{\rho}\left(\lambda_{1}, \lambda_{2}\right)=\sum_{l=1}^{n}\left(\frac{y_{1 l}^{\left(\lambda_{1}\right)}-\hat{\mu}_{1 n}\left(\lambda_{1}\right)}{\hat{\sigma}_{1 n}\left(\lambda_{1}\right)}\right)\left(\frac{y_{2 l}^{\left(\lambda_{2}\right)}-\hat{\mu}_{2 n}\left(\lambda_{2}\right)}{\hat{\sigma}_{2 n}\left(\lambda_{2}\right)}\right)
\end{gathered}
$$

respectively. Substitution of the these maximum likelihood estimates into the log-likelihood function given by (2.1.12) yields, apart from constant term

$$
\log L_{n}(\boldsymbol{\theta})=-\frac{n}{2}\left\{\log \hat{\sigma}_{1 n}^{2}\left(\lambda_{1}\right)+\log \hat{\sigma}_{2 n}^{2}\left(\lambda_{2}\right)+\log \left(1-\hat{\rho}^{2}\left(\lambda_{1}, \lambda_{2}\right)\right)\right\}
$$


Inferences Based on the Grouped Observations from The Bivariate Power-Normal Distribution

$$
+\left(\lambda_{1}-1\right) \sum_{l=1}^{n} \log y_{1 l}+\left(\lambda_{2}-1\right) \sum_{l=1}^{n} \log y_{2 l}
$$

The maximum likelihood estimates $\hat{\lambda}_{1 n}$ and $\hat{\lambda}_{2 n}$ are the values of the transforming parameter $\lambda_{1}$ and $\lambda_{2}$ for which the maximized log-likelihood is a maximum. Furthermore, the substitution of $\hat{\lambda}_{1 n}$ and $\hat{\lambda}_{2 n}$ into $\hat{\mu}_{1 n}\left(\lambda_{1}\right), \hat{\mu}_{2 n}\left(\lambda_{2}\right), \hat{\sigma}_{1 n}^{2}\left(\lambda_{1 n}\right), \hat{\sigma}_{2 n}^{2}\left(\lambda_{2 n}\right)$ and $\hat{\rho}_{n}\left(\lambda_{1 n}, \lambda_{2 n}\right)$ yields, the maximum likelihood estimates $\hat{\mu}_{1 n}\left(\hat{\lambda}_{1 n}\right), \hat{\mu}_{2 n}\left(\hat{\lambda}_{2 n}\right), \hat{\sigma}_{1 n}^{2}\left(\hat{\lambda}_{1 n}\right), \hat{\sigma}_{2 n}^{2}\left(\hat{\lambda}_{2 n}\right)$ and $\hat{\rho}_{n}\left(\hat{\lambda}_{1 n}, \hat{\lambda}_{2 n}\right)$ respectively.

\subsection{The Procedure of Fitting the BPND to the Grouped Observations}

In this section, the procedure of fitting the BPND to two variables, in which observations are grouped into several intervals, is developed.

Let the observations be grouped into $k \times l$ cells, specified beforehand and determined by $k(k \geq 3)$ interval with $I_{11}=\left[y_{10}, y_{11}\right), I_{12}=\left[y_{11}, y_{12}\right), \cdots, I_{1 k}=\left[y_{1 k-1}, y_{1 k}\right)$ on $Y_{1}$, and by $l(l \geq 3)$ interval with $I_{21}=\left[y_{20}, y_{21}\right), I_{22}=\left[y_{21}, y_{22}\right), \cdots, I_{2 l}=\left[y_{2 l-1}, y_{2 l}\right)$ on $Y_{2}$, where $0=y_{10}<y_{11}<\cdots<y_{1 k-1}<y_{1 k}=\infty$ and $0=y_{20}<y_{21}<\cdots<y_{2 k-1}<y_{2 k}=\infty$. Then, the frequency of the observations lying in the $i j$ th cell is denoted by $n_{i j}$, and is given by the two-way frequency table shown as Table 2.2.1. In general, it is called a correlation table (Kendall and Buckland, 1982: Kitagawa and Inaba, 1979).

Table 2.2.1. Correlation table for two set of observations $Y_{1}$ and $Y_{2}$

\begin{tabular}{cc|cccccccc}
\hline & & \multicolumn{5}{c}{$Y_{1}$} & & & \\
& & {$\left[y_{10}, y_{11}\right)$} & {$\left[y_{11}, y_{12}\right)$} & $\cdots$ & {$\left[y_{1 i-1}, y_{1 i}\right)$} & $\cdots$ & {$\left[y_{1 k-1}, y_{1 k}\right)$} & Total \\
\hline \multirow{4}{*}{$Y_{2}$} & {$\left[y_{20}, y_{21}\right)$} & $n_{11}$ & $n_{21}$ & $\cdots$ & $n_{i 1}$ & $\cdots$ & $n_{k 1}$ & $n_{\cdot 1}$ \\
& $\left(y_{21}, y_{22}\right)$ & $n_{12}$ & $n_{22}$ & $\cdots$ & $n_{i 2}$ & $\cdots$ & $n_{k 2}$ & $n_{\cdot 2}$ \\
& $\vdots$ & $\vdots$ & $\vdots$ & & $\vdots$ & & $\vdots$ & $\vdots$ \\
& $\left(y_{2 j-1}, y_{2 j}\right)$ & $n_{1 j}$ & $n_{2 j}$ & $\cdots$ & $n_{i j}$ & $\cdots$ & $n_{k j}$ & $n_{\cdot j}$ \\
& $\vdots$ & $\vdots$ & $\vdots$ & & $\vdots$ & & $\vdots$ & $\vdots$ \\
& {$\left[y_{2 l-1}, y_{2 l}\right)$} & $n_{1 l}$ & $n_{2 l}$ & $\cdots$ & $n_{i l}$ & $\cdots$ & $n_{k l}$ & $n_{\cdot l}$ \\
\hline Total & $n_{1 .}$ & $n_{2 .}$ & $\cdots$ & $n_{i}$ & $\cdots$ & $n_{k}$. & $n$ \\
\hline
\end{tabular}

Therefore, for grouped observations shown as correlation table in Table 2.2.1, the loglikelihood function for a sample size $n=\sum_{i=1}^{k} \sum_{j=1}^{l} n_{i j}$, can be written as

$$
\log L_{n}(\boldsymbol{\theta})=\log n !-\sum_{i=1}^{k} \sum_{j=1}^{l} \log n_{i j}+\sum_{i=1}^{k} \sum_{j=1}^{l} n_{i j} \log p_{\mathrm{BP}_{i j}}(\boldsymbol{\theta})
$$

where $p_{\mathrm{BP}_{i j}}(\boldsymbol{\theta})$ is the probability based on the BPND and is given by

$$
\begin{aligned}
& p_{\mathrm{BP}_{i j}}(\boldsymbol{\theta})=\frac{1}{A(\boldsymbol{\lambda}, \boldsymbol{\mu}, \boldsymbol{\Sigma})}\left\{\Phi_{2}\left(z_{1 i}, z_{2 j}\right)+\Phi_{2}\left(z_{1 i-1}, z_{2 j-1}\right)-\Phi_{2}\left(z_{1 i}, z_{2 j-1}\right)-\Phi_{2}\left(z_{1 i-1}, z_{2 j}\right)\right\} \\
& \text { with } z_{1 i}=\left(y_{1 i}^{\left(\lambda_{1}\right)}-\mu_{1}\right) / \sigma_{1} \text { and } z_{2 i}=\left(y_{2 i}^{\left(\lambda_{2}\right)}-\mu_{2}\right) / \sigma_{2} .
\end{aligned}
$$


The maximum likelihood estimates $\hat{\lambda}_{1}, \hat{\lambda}_{2}, \hat{\mu}_{1}, \hat{\mu}_{2}, \hat{\sigma}_{1}, \hat{\sigma}_{1}$ and $\hat{\rho}$ of $\lambda_{1}, \lambda_{2}, \mu_{1}, \mu_{2}$, $\sigma_{1}, \sigma_{2}$ and $\rho$ can be obtained by maximizing the log-likelihood function (2.2.1). However, the log-likelihood function (2.2.4) are non-linear with respect to transforming parameters $\lambda_{1}$ and $\lambda_{2}$, so the maximum likelihood estimates $\hat{\lambda}_{1}, \hat{\lambda}_{2}, \hat{\mu}_{1}, \hat{\mu}_{2}, \hat{\sigma}_{1}, \hat{\sigma}_{1}$ and $\hat{\rho}$ can not be presented explicitly. Even when $\lambda_{1}$ and $\lambda_{2}$ are given as $\lambda_{1}^{*}$ and $\lambda_{2}^{*}$, or $A(\boldsymbol{\lambda}, \boldsymbol{\mu}, \boldsymbol{\Sigma})=1$ is assumed, the maximum likelihood estimates can not be presented explicitly as (2.1.3). Actually, the two-stage procedure is employed to obtain the maximum likelihood estimates; first for fixed $\lambda_{1}$ and $\lambda_{2}$, the $\log$-likelihood function $\log L_{n}(\boldsymbol{\theta})$ is maximized over the values of the remaining parameters, $\mu_{1}, \mu_{2}, \sigma_{1}, \sigma_{2}$ and $\rho$. Then, global maximum of is searched on a grid of values of $\lambda_{1}$ and $\lambda_{2}$. This two-stage procedure will produce the desired value provided the assumptions of Appendices hold and the matrix $\left.\nabla_{\left(\mu_{1}, \mu_{2}, \sigma_{1}, \sigma_{2}, \rho\right)}^{2} \log L_{n}(\boldsymbol{\theta})\right|_{\theta=\hat{\theta}_{n}}$ is nonsingular. The approximate $100(1-\alpha) \%$ confidence region for these estimates are then found from those values for which $2\left\{\log L_{n}(\hat{\boldsymbol{\theta}})-\log L_{n}(\boldsymbol{\theta})\right\}>\chi_{7}^{2}(\alpha)$. The strong consistency and the asymptotic normality of the maximum likelihood estimators $\hat{\lambda}_{1}, \hat{\lambda}_{2}$, $\hat{\mu}_{1}, \hat{\mu}_{2}, \hat{\sigma}_{1}, \hat{\sigma}_{1}$ and $\hat{\rho}$ can be established under certain conditions. The proofs are given in Appendices.

\section{Some Examples}

To have a concrete and practical grasp of the aim in this paper, and make the impression of them clear, the performance of fitting the BPND to the grouped observations is numerically examined through some examples cited from the published literatures. The contents of five examples taken up for discussion are shown in Table 3.1. To evaluate the bivariate normality of the simultaneous power-transformed observations, we adopted the following three tests.

Test 1 : test whether third and fourth cumulants equal to zero or not (Dahiya and Gurland, 1973: Takeuchi, 1974)

Test 2 : assess multi-dimensional skewness and assess multi-dimensional kurtosis (Mardia, 1970)

Test 3 : assess the size of sample which falls into the each of four quadrants of two-dimensional coordinates (Takeuchi, 1974: Shibata, 1981)

Then, the results of fitting the BPND to these five examples were summarized as the values of maximum likelihood estimates, $\hat{\lambda}_{1}, \hat{\lambda}_{2}, \hat{\mu}_{1}, \hat{\mu}_{2}, \hat{\sigma}_{1}, \hat{\sigma}_{2}$, and $\hat{\rho}$ of $\lambda_{1}, \lambda_{2}, \mu_{1}, \mu_{2}, \sigma_{1}$, $\sigma_{2}$ and $\rho$, the three test above and its p-value together with results of fitting of the PND. The followings are the consideration processes for these results.

Example 1: Cramér (1974) has considered the relationship between the age of father and mother of 475,322 boys of live born children in Norway during the period 1871-1900, in which the results of observations are shown as the frequencies in several groups of the age of father and mother. The results of fitting the BPND to these observations setting the age of father as $Y_{1}$ and the age of mother as $Y_{2}$ are shown in Table 3.2. The transforming parameter for the age of father was estimated as -0.0630 , which was close to zero, whereas for the age of mother, as $\mathbf{0 . 4 3 3 0}$. These transforming parameters suggest that both the observations of the age of father and mother have a log-normal and exponential respectively. 
Inferences Based on the Grouped Observations from The Bivariate Power-Normal Distribution

Table 3.1. $\quad$ List of Examples

\begin{tabular}{c|l|l}
\hline No. & \multicolumn{1}{|c|}{ Literature } & \multicolumn{1}{c}{ Contents } \\
\hline 1 & Cramer (1974) & $\begin{array}{l}\text { The frequencies in several groups of age of parents for } \\
475,322 \text { boy children in Norway during the period 1871- } \\
1900 .\end{array}$ \\
\hline 2 & Holmes (1974) & $\begin{array}{l}\text { The frequencies in several groups of total miles driven and } \\
\text { family income for 4012 families (car owners) during 1973. }\end{array}$ \\
\hline 4 & $\begin{array}{l}\text { Wardia (1970) } \\
\text { Stuart and Ord (1986) }\end{array}$ & $\begin{array}{l}\text { The frequencies in the number of employers and the num- } \\
\text { ber of unemployment spells during the period 1974-1984 } \\
\text { for 1962 individuals, provided by German socio-economic } \\
\text { panel (1994) }\end{array}$ \\
\hline 5 & Stuart and Ord (1986) \\
both length and breadth.
\end{tabular}

Table 3.2. The results of fitting of the BPND (Example 1)

\begin{tabular}{|c|c|c|c|c|c|c|}
\hline & \multirow{2}{*}{\multicolumn{2}{|c|}{$\begin{array}{c}\text { Original } \\
\text { (Untransformed) }\end{array}$}} & \multirow{2}{*}{\multicolumn{2}{|c|}{$\begin{array}{c}\text { BPND } \\
\text { (Transformed) }\end{array}$}} & \multicolumn{2}{|c|}{ PND } \\
\hline & & & & & Age of father & Age of mother \\
\hline \multicolumn{7}{|c|}{ Maximum likelihood and estimate } \\
\hline $\begin{array}{l}\text { Maximum } \\
\text { log-likelihood }\end{array}$ & \multicolumn{2}{|c|}{-} & \multicolumn{2}{|c|}{-1608753.999} & -2282.3949 & -6816.4912 \\
\hline$\hat{\lambda}_{1}$ & & - & & -0.0630 & 0.1204 & - \\
\hline$\hat{\lambda}_{2}$ & & - & & 0.433 & - & 0.3613 \\
\hline$\hat{\mu}_{1}$ & & 35.6991 & & 3.1749 & 4.3941 & \\
\hline$\hat{\mu}_{1}^{*}$ & \multirow{3}{*}{\multicolumn{2}{|c|}{32.1275}} & & 34.5489 & 34.0128 & - \\
\hline$\hat{\mu}_{2}$ & & & & 8.0022 & & 6.8835 \\
\hline$\hat{\mu}_{2}^{*}$ & & & & 31.6759 & - & 31.7219 \\
\hline$\hat{\sigma}_{1}$ & \multicolumn{2}{|r|}{8.5334} & & 0.1904 & 0.3310 & - \\
\hline$\hat{\sigma}_{2}$ & \multicolumn{2}{|r|}{6.7001} & & 0.9445 & - & 0.7266 \\
\hline$\hat{\rho}$ & \multicolumn{2}{|c|}{0.6183} & & 0.6264 & - & - \\
\hline \multirow{5}{*}{ Test 1} & \multicolumn{2}{|c|}{ Performance } & & & & \\
\hline & $\hat{\gamma}_{12}$ & 0.1610 & $\hat{\gamma}_{12}$ & 0.0421 & & \\
\hline & $\mathrm{p}$-value & near 0 & $\mathrm{p}$-value & near 0 & & \\
\hline & $\hat{\gamma}_{21}$ & 0.1971 & $\hat{\gamma}_{21}$ & 0.0077 & & \\
\hline & $\mathrm{p}$-value & near 0 & $\mathrm{p}$-value & 0.0024 & & \\
\hline \multirow{4}{*}{ Test2 } & Skewness & 0.0002 & Skewness & 0.0001 & & \\
\hline & $\mathrm{p}$-value & near 0 & $\mathrm{p}$-value & 0.0504 & & \\
\hline & Kurtosis & 9.3914 & Kurtosis & 8.1434 & & \\
\hline & $\mathrm{p}$-value & near 0 & $\mathrm{p}$-value & near 0 & & \\
\hline \multirow{2}{*}{ Test3 } & $\chi^{2}$ & 48140.7147 & $\chi^{2}$ & 47618.3182 & & \\
\hline & $\mathrm{p}$-value & near 0 & $\mathrm{p}$-value & near 0 & & \\
\hline
\end{tabular}


These results agree with the results for fitting the PND to each of these two variables (The transforming parameters were estimated as 0.1204 and 0.3613 ). For the estimated values of these two transforming parameters, $34.5489,8.0022,0.1904,0.9445$ were obtained. The values $\hat{\mu}_{1}^{*}\left(\hat{\lambda}_{1}\right)$ and $\hat{\mu}_{2}^{*}\left(\hat{\lambda}_{2}\right)$ of back-transformed $\hat{\mu}_{1}\left(\hat{\lambda}_{1}\right)$ and $\hat{\mu}_{2}\left(\hat{\lambda}_{2}\right)$ to the original scale were 34.5489 and 31.6789 , both of which were $\hat{\mu}_{1}(1)=35.6991$ and $\hat{\mu}_{2}(1)=32.1275$ or lower. Also, $\hat{\rho}\left(\hat{\lambda}_{1}, \hat{\lambda}_{2}\right)$ was estimated as 0.6264 , which was $\hat{\rho}(1,1)=0.6183$ or slightly larger on the original observations.

The results of the three tests shown in Table 3.2, the power-transformed observations satisfied more a bivariate normality than the original observations.

Example 2: Homles (1974) has considered the relationship between family income and the amount of money spent on gasoline based on the observed value of them for 4,012 families (car owner) during 1973. Thus, as the amount of money spent on gasoline could not be directly observed, he used the total miles driven instead of the amount of money. The results of observations are shown as the frequencies in several groups of total miles driven and family income. The results of fitting the BPND to these observations setting family income as $Y_{1}$ and total miles driven as $Y_{2}$ are shown in Table 3.3. The transforming parameter for family income was estimated as 0.3600 , whereas for total miles driven, as 0.3550 . These estimates suggest that both the observations of family income and total miles driven have an exponential distribution. These results agree with the results for fitting the PND to each of these two variables (The transforming parameters were estimated as 0.3504 and 0.4453). For the estimated values of these two transforming parameters, $\hat{\mu}_{1}\left(\hat{\lambda}_{1}\right)=76.3190$, $\hat{\mu}_{2}\left(\hat{\lambda}_{2}\right)=4.2016, \hat{\sigma}_{1}\left(\hat{\lambda}_{1}\right)=17.3144$, and $\hat{\sigma}_{2}\left(\hat{\lambda}_{2}\right)=1.7868$ were obtained. The values $\hat{\mu}_{1}^{*}\left(\hat{\lambda}_{1}\right)$ and $\hat{\mu}_{2}^{*}\left(\hat{\lambda}_{2}\right)$ of back-transformed $\hat{\mu}_{1}^{*}\left(\hat{\lambda}_{1}\right)$ and $\hat{\mu}_{2}^{*}\left(\hat{\lambda}_{2}\right)$ to the original scale were 10969.0682 and 13.0865 , both of which were $\hat{\mu}_{1}(1)=12259.5713$ and $\hat{\mu}_{2}(1)=15.2430$ or lower. Also, $\hat{\rho}\left(\hat{\lambda}_{1}, \hat{\lambda}_{2}\right)$ was estimated as 0.4381 , which was 0.4009 or slightly larger on the original scale. The results of the three tests shown in Table 3.3, the power-transformed observations satisfied more a bivariate normality than the original observations.

Example 3: Winkelmann (1994) has considered the relationship between the number of employers and the number of unemployment spells during the period 1974-1984 for 1962 individuals, provided by German Socio-economic panel (SOEP), in which the results of the observations are shown as the frequencies classified by the number of employers and the number of unemployment spells. And using the information on the number of employers and the number of unemployment spells, he investigated the direct job change. The results of fitting the BPND to these observations setting the number of employers as $Y_{1}$ and the number of unemployment spells as $Y_{2}$ are shown in Table 3.4. The transforming parameter for the number of employers was estimated as -0.1270 , whereas for the number of employment spells, as -1.6470 . These estimates suggest that both the observations of employers and unemployment spells both have an L-shaped distribution. However, the transforming parameters in fitting the PND to each of the number of employers and the number of unemployment spells were estimated as 0.3011 and 0.1204 , which were near zero and suggest log-normal. The results of fitting the BPND to this example did not agree with those of the PND. For the estimated values of these two transforming parameters, $\hat{\mu}_{1}\left(\hat{\lambda}_{1}\right)=-0.8054$, $\hat{\mu}_{2}\left(\hat{\lambda}_{2}\right)=-1.7013, \hat{\sigma}_{1}\left(\hat{\lambda}_{1}\right)=0.7623$, and $\hat{\sigma}_{2}\left(\hat{\lambda}_{2}\right)=0.7963$ were obtained. The values $\hat{\mu}_{1}^{*}\left(\hat{\lambda}_{1}\right)$ and $\hat{\mu}_{2}^{*}\left(\hat{\lambda}_{2}\right)$ of back-transformed $\hat{\mu}_{1}\left(\hat{\lambda}_{1}\right)$ and $\hat{\mu}_{2}\left(\hat{\lambda}_{2}\right)$ to the original scale were 0.4645 and 0.4445. Then, $\hat{\mu}_{1}^{*}\left(\hat{\lambda}_{1}\right)$ was $\hat{\mu}_{1}(1)=0.5395$ or lower, whereas $\hat{\mu}_{2}\left(\hat{\lambda}_{2}\right)$ was $\hat{\mu}_{2}(1)=0.3736$ or 
Inferences Based on the Grouped Observations from The Bivariate Power-Normal Distribution

Table 3.3. The results of fitting of the BPND (Example 2)

\begin{tabular}{|c|c|c|c|c|c|c|}
\hline & \multirow{2}{*}{\multicolumn{2}{|c|}{$\begin{array}{c}\text { Original } \\
\text { (Untransformed) }\end{array}$}} & \multirow{2}{*}{\multicolumn{2}{|c|}{$\begin{array}{c}\text { BPND } \\
\text { (Transformed) }\end{array}$}} & \multicolumn{2}{|c|}{ PND } \\
\hline & & & & & Family income & Miles driven \\
\hline \multicolumn{7}{|c|}{ Maximum likelihood and estimate } \\
\hline $\begin{array}{l}\text { Maximum } \\
\text { log-likelihood }\end{array}$ & \multicolumn{2}{|c|}{-} & \multicolumn{2}{|c|}{-16621.4776} & -45.1614 & -51.0135 \\
\hline$\hat{\lambda}_{1}$ & & - & & 0.3600 & 0.3504 & - \\
\hline$\hat{\lambda}_{2}$ & & - & & 0.3550 & - & 0.4453 \\
\hline$\hat{\hat{\mu}_{1}}$ & \multirow{4}{*}{\multicolumn{2}{|c|}{12259.5713}} & & 76.3190 & 71.4135 & \\
\hline$\hat{\mu}_{1}^{*}$ & & & & $(10969.0682)$ & $(11062.5581)$ & - \\
\hline$\hat{\mu}_{2}$ & & & & 4.2016 & & 4.6688 \\
\hline$\hat{\mu}_{2}^{*}$ & & & & $(13.0865)$ & $\quad-$ & $(13.2120)$ \\
\hline$\hat{\sigma}_{1}$ & \multicolumn{2}{|r|}{6802.4333} & & 17.3144 & 17.0563 & - \\
\hline$\hat{\sigma}_{2}$ & \multicolumn{2}{|r|}{10.1955} & & 1.7868 & - & 2.4593 \\
\hline$\hat{\rho}$ & \multicolumn{2}{|c|}{0.4009} & & 0.4381 & - & - \\
\hline \multirow{5}{*}{ Test1 } & \multicolumn{2}{|c|}{ Performance } & & & & \\
\hline & $\hat{\gamma}_{12}$ & 0.1188 & $\hat{\gamma}_{12}$ & 0.0450 & & \\
\hline & $\mathrm{p}$-value & near 0 & $\mathrm{p}$-value & near 0 & & \\
\hline & $\hat{\gamma}_{21}$ & 0.1261 & $\hat{\gamma}_{21}$ & 0.0456 & & \\
\hline & $\mathrm{p}$-value & near 0 & $\mathrm{p}$-value & 0.0407 & & \\
\hline \multirow{4}{*}{ Test2 } & Skewness & 0.0097 & Skewness & 0.0075 & & \\
\hline & $\mathrm{p}$-value & 0.1640 & p-value & 0.2831 & & \\
\hline & Kurtosis & 7.5353 & Kurtosis & 6.7365 & & \\
\hline & $\mathrm{p}$-value & 0.9999 & $\mathrm{p}$-value & 0.9999 & & \\
\hline \multirow{2}{*}{ Test3 } & $\chi^{2}$ & 705.9977 & $\chi^{2}$ & 329.8819 & & \\
\hline & $\mathrm{p}$-value & near 0 & p-value & near 0 & & \\
\hline
\end{tabular}


Table 3.4. The results of fitting of the BPND (Example 3)

\begin{tabular}{|c|c|c|c|c|c|c|}
\hline & \multirow{2}{*}{\multicolumn{2}{|c|}{$\begin{array}{c}\text { Original } \\
\text { (Untransformed) }\end{array}$}} & \multirow{2}{*}{\multicolumn{2}{|c|}{$\begin{array}{c}\text { BPND } \\
\text { (Transformed) }\end{array}$}} & \multicolumn{2}{|c|}{ PND } \\
\hline & & & & & Employers & Uneployment \\
\hline \multicolumn{7}{|c|}{ Maximum likelihood and estimate } \\
\hline $\begin{array}{l}\text { Maximum log- } \\
\text { likelihood }\end{array}$ & & - & & -3642.3400 & -24.1869 & -31.2127 \\
\hline$\hat{\lambda}_{1}$ & & - & & -0.1270 & 0.3011 & - \\
\hline$\hat{\lambda}_{2}$ & & - & & -1.6470 & - & 0.1204 \\
\hline$\hat{\mu}_{1}$ & & 0.5395 & & -0.8054 & -1.2943 & \\
\hline$\hat{\mu}_{1}^{*}$ & & & & $(0.4645)$ & $(0.1940)$ & - \\
\hline$\hat{\mu}_{2}$ & & 0.3736 & & -1.7013 & & -2.0413 \\
\hline$\hat{\mu}_{2}^{*}$ & & & & $(0.4445)$ & - & $(0.0961)$ \\
\hline$\hat{\sigma}_{1}$ & & 1.0828 & & 0.7623 & 1.4323 & - \\
\hline$\hat{\sigma}_{2}$ & & 1.1049 & & 0.7963 & - & 1.6545 \\
\hline$\hat{\hat{\rho}}$ & & 0.0501 & & 0.1096 & - & - \\
\hline \multirow{4}{*}{ Test1 } & Perfo & nance & & & & \\
\hline & $\begin{array}{r}\hat{\gamma}_{12} \\
\text { p-value }\end{array}$ & $\begin{array}{l}0.1341 \\
\text { near } 0\end{array}$ & $\begin{array}{r}\hat{\gamma}_{12} \\
\text { p-value }\end{array}$ & $\begin{array}{l}0.1603 \\
\text { near } 0\end{array}$ & & \\
\hline & $\hat{\gamma}_{21}$ & 0.3262 & $\hat{\gamma}_{21}$ & 0.2013 & & \\
\hline & $\mathrm{p}$-value & near 0 & $\mathrm{p}$-value & 0.0021 & & \\
\hline \multirow{3}{*}{ Test2 } & Skewness & 2.5966 & Skewness & 0.0516 & & \\
\hline & p-value & 0.1640 & $\mathrm{p}$-value & 0.2831 & & \\
\hline & Kurtosis & 70.4191 & Kurtosis & 11.3122 & & \\
\hline \multirow{2}{*}{ Test3 } & $\frac{p-v a i u e}{\chi^{2}}$ & 2827.9281 & $\frac{p-v a t e}{\chi^{2}}$ & $2765: 4151$ & & \\
\hline & $\mathrm{p}$-value & near 0 & $\mathrm{p}$-value & near 0 & & \\
\hline
\end{tabular}


larger. Also, $\hat{\rho}\left(\hat{\lambda}_{1}, \hat{\lambda}_{2}\right)$ was estimated as 0.1096 , which was $\hat{\rho}(1,1)=0.0501$ or slightly larger on the original scale.

The results of the three tests shown in Table 3.4, the power-transformed observations satisfied more a bivariate normality than the original observations.

Example 4: Mardia (1970) and Kendall and Stuart (1977) have considered the relationship between length and breadth of 9,440 beans, in which the results of the observations are shown as the frequencies in several groups of the length and the breadth. The results of fitting the BPND to these observations setting length as $Y_{1}$ and breadth as $Y_{2}$ are shown in Table 3.5. The transforming parameter for the length was estimated as 2.3500 , whereas for the breadth, as 1.9260. These estimates suggest that both observations of the length and the breadth both have a J-shaped distribution. These results agree with the results for fitting the PND

Table 3.5. The results of fitting of the BPND (Example 4)

\begin{tabular}{|c|c|c|c|c|c|c|}
\hline & \multirow{2}{*}{\multicolumn{2}{|c|}{$\begin{array}{c}\text { Original } \\
\text { (Untransformed) }\end{array}$}} & \multirow{2}{*}{\multicolumn{2}{|c|}{$\begin{array}{c}\text { BPND } \\
\text { (Transformed) }\end{array}$}} & \multicolumn{2}{|c|}{ PND } \\
\hline & & & & & Lengths & Breadth \\
\hline \multicolumn{7}{|c|}{ Maximum likelihood and estimate } \\
\hline $\begin{array}{l}\text { Maximum log- } \\
\text { likelihood }\end{array}$ & & - & & -30926.9648 & -185.7441 & -73.1354 \\
\hline$\hat{\lambda}_{1}$ & & - & & 2.3500 & 3.0319 & \\
\hline$\hat{\lambda}_{2}$ & & - & & 1.9260 & - & 3.1300 \\
\hline$\hat{\mu}_{1}$ & & 14.4035 & & 225.6732 & 1033.7271 & \\
\hline$\hat{\mu}_{1}^{*}$ & & & & $(14.4454)$ & $(14.4599)$ & - \\
\hline$\hat{\mu}_{2}$ & & 7.9724 & & 27.8370 & & 172.8152 \\
\hline$\hat{\mu}_{2}^{*}$ & & & & $(7.9804)$ & - & $(7.9873)$ \\
\hline$\hat{\sigma}_{1}$ & & 0.9166 & & 32.4748 & 184.0468 & - \\
\hline$\hat{\sigma}_{2}$ & & 0.3493 & & 2.3710 & - & 22.0982 \\
\hline$\hat{\rho}$ & & 0.7590 & & 0.7562 & - & - \\
\hline \multirow{3}{*}{ Test1 } & Perfor & nance & & & & \\
\hline & $\begin{array}{r}\hat{\gamma}_{12} \\
\text { p-value }\end{array}$ & $\begin{array}{l}0.4605 \\
\text { near } 0\end{array}$ & $\begin{array}{r}\hat{\gamma}_{12} \\
\text { p-value }\end{array}$ & $\begin{array}{l}0.3076 \\
\text { near } 0\end{array}$ & & \\
\hline & $\begin{array}{r}\hat{\gamma}_{21} \\
\text { p-value }\end{array}$ & $\begin{array}{l}0.6177 \\
\text { near } 0\end{array}$ & $\begin{array}{r}\hat{\gamma}_{21} \\
\text { p-value }\end{array}$ & $\begin{array}{l}0.3986 \\
\text { near } 0\end{array}$ & & \\
\hline \multirow{4}{*}{ Test2 } & Skewness & 0.0105 & Skewness & 0.0074 & & \\
\hline & p-value & 0.0026 & p-value & 0.0220 & & \\
\hline & Kurtosis & 10.2394 & Kurtosis & 9.1569 & & \\
\hline & $\mathrm{p}$-value & near 0 & p-value & near 0 & & \\
\hline \multirow{2}{*}{ Test3 } & $\chi^{2}$ & 1380.0420 & $\chi^{2}$ & 1379.2665 & & \\
\hline & $\mathrm{p}$-value & near 0 & $\mathrm{p}$-value & near 0 & & \\
\hline
\end{tabular}

to each of these two variables (The transforming parameters were estimated as 3.0319 and $3.1300)$. For the estimated values of these two transforming parameters, $\hat{\mu}_{1}\left(\hat{\lambda}_{1}\right)=225.6732$, $\hat{\mu}_{2}\left(\hat{\lambda}_{2}\right)=27.8370, \hat{\sigma}_{1}\left(\hat{\lambda}_{1}\right)=32.4748$, and $\hat{\sigma}_{2}\left(\hat{\lambda}_{2}\right)=2.3710$ were obtained. The values $\hat{\iota}_{1}^{*}\left(\hat{\lambda}_{1}\right)$ and $\hat{\mu}_{2}^{*}\left(\hat{\lambda}_{2}\right)$ of back-transformed $\hat{\mu}_{1}\left(\hat{\lambda}_{1}\right)$ and $\hat{\mu}_{2}\left(\hat{\lambda}_{2}\right)$ to the original scale were 14.4454 and 7.9804 , in which both were close to $\hat{\mu}_{1}\left(\hat{\lambda}_{1}\right)=14.4035$ and $\hat{\mu}_{1}\left(\hat{\lambda}_{2}\right)=7.9804$. Also, $\hat{\rho}\left(\hat{\lambda}_{1}, \hat{\lambda}_{2}\right)$ was estimated as 0.7562 , which was 0.7590 or slightly smaller on the original scale. 
The results of the three tests shown in Table 3.5, the power-transformed observations satisfied more a bivariate normality than the original observations.

Example 5: Stuart and Ord (1986) have considered the relationship between the number of newspapers read and the number of newspapers just looked at, students of the University of London, in which the results of the observations are shown as the frequencies of the number of newspapers read and the number of newspapers just looked at. The results of fitting the BPND to these observations setting the newspaper read as $Y_{1}$ and the newspaper just looked at as $Y_{2}$ are shown in Table 3.6. The transforming parameter for the newspapers read was estimated as 0.4720 , whereas for the newspapers just looked at, as 0.7080 . These estimates suggest that the observations of both the newspaper read and just looked at have an exponential distribution. These results agree with the results for fitting the PND to

Table 3.6. The results of fitting of the BPND (Example 5)

\begin{tabular}{|c|c|c|c|c|c|c|}
\hline & \multirow{2}{*}{\multicolumn{2}{|c|}{$\begin{array}{c}\text { Original } \\
\text { (Untransformed) }\end{array}$}} & \multirow{2}{*}{\multicolumn{2}{|c|}{$\begin{array}{c}\text { BPND } \\
\text { (Transformed) }\end{array}$}} & \multicolumn{2}{|r|}{ PND } \\
\hline & & & & & Read & Just looked at \\
\hline \multicolumn{7}{|c|}{ Maximum likelihood and estimate } \\
\hline $\begin{array}{l}\text { Maximum log- } \\
\text { likelihood }\end{array}$ & - & & & -2264.2991 & -15.0338 & -13.2485 \\
\hline$\hat{\lambda}_{1}$ & & - & & 0.4720 & 0.4818 & - \\
\hline$\hat{\lambda}_{2}$ & & - & & -0.7080 & - & 0.6022 \\
\hline$\hat{\mu}_{1}$ & & 0.9777 & & -0.1961 & 0.0779 & \\
\hline$\hat{\mu}_{1}^{*}$ & & & & $(0.8140)$ & (1.0795) & - \\
\hline$\hat{\mu}_{2}$ & & 1.2327 & & 0.1369 & & -0.2971 \\
\hline$\hat{\mu}_{2}^{*}$ & & & & (1.1396) & - & $(0.7208)$ \\
\hline$\hat{\sigma}_{1}$ & & 1.0707 & & 0.9087 & 0.8009 & - \\
\hline$\hat{\sigma}_{2}$ & & 0.9298 & & 0.8377 & - & 1.1460 \\
\hline$\hat{\rho}$ & & 0.0936 & & 0.0935 & - & - \\
\hline \multirow{5}{*}{ Test1 } & Perfor & nance & & & & \\
\hline & $\hat{\gamma}_{12}$ & 0.0823 & $\hat{\gamma}_{12}$ & 0.0679 & & \\
\hline & $\mathrm{p}$-value & 0.0419 & $\mathrm{p}$-value & 0.0769 & & \\
\hline & $\hat{\gamma}_{21}$ & 0.0888 & $\hat{\gamma}_{21}$ & 0.0828 & & \\
\hline & $\mathrm{p}$-value & 0.0014 & p-value & 0.0434 & & \\
\hline \multirow{4}{*}{ Test2 } & Skewness & 0.1187 & Skewness & 0.0657 & & \\
\hline & $\mathrm{p}$-value & 0.0311 & p-value & 0.0411 & & \\
\hline & Kurtosis & 10.6200 & Kurtosis & 8.2923 & & \\
\hline & p-value & near 0 & p-value & 0.1368 & & \\
\hline \multirow{2}{*}{ Test3 } & $\chi^{2}$ & 377.3460 & $\chi^{2}$ & 377.4072 & & \\
\hline & p-value & near 0 & $\mathrm{p}$-value & near 0 & & \\
\hline
\end{tabular}

each of these two variables (The transforming parameters were estimated as 0.4818 and $0.6022)$. For the estimated values of these two transforming parameters, $\hat{\mu}_{1}\left(\hat{\lambda}_{1}\right)=-0.1961$, $\hat{\mu}_{2}\left(\hat{\lambda}_{2}\right)=0.1369, \hat{\sigma}_{1}\left(\hat{\lambda}_{1}\right)=0.9087$, and $\hat{\sigma}_{2}\left(\hat{\lambda}_{2}\right)=0.8377$ were obtained. The values $\hat{\mu}_{1}^{*}\left(\hat{\lambda}_{1}\right)$ and $\hat{\mu}_{2}^{*}\left(\hat{\lambda}_{2}\right)$ of back-transformed $\hat{\mu}_{1}\left(\hat{\lambda}_{1}\right)$ and $\hat{\mu}_{2}\left(\hat{\lambda}_{2}\right)$ to the original scale were 0.8140 and 1.1396, both of which were $\hat{\mu}_{1}(1)=0.9777$ and $\hat{\mu}_{2}(1)=1.2327$ or lower. Also, $\hat{\rho}\left(\hat{\lambda}_{1}, \hat{\lambda}_{2}\right)$ was 
estimated as 0.0935 , which was close to 0.0936 on the original scale.

The results of the three tests shown in Table 3.6, the power-transformed observations satisfied more a bivariate normality than the original observations.

These results of considering the five examples show bivariate power-normal distribution will be helpful to "regularize" the observations even when strict bivariate normality was not achieved. Also, the correlation coefficient for power-transformed observations increases more than the original observations. For ungrouped observations, if $(X, Y)$ are jointly distributed in the bivariate normal distribution with correlation $\rho$, and transformation is made to $X^{*}=X^{*}(X)$ and $Y^{*}=Y^{*}(Y)$ with $\mathrm{E}_{X}\left[X^{* 2}\right]$ and $\mathrm{E}_{Y}\left[Y^{* 2}\right]$ both finite, then the correlation of the new variables $X^{*}$ and $Y^{*}$ is less in absolute value than $\rho$. Namely, for ungrouped observations, transforming so that the bivariate normality is more nearly satisfied should increase the correlation coefficient in absolute value. This suggests that the same may be true in grouped observations. As an example, the distributions of the original and the transformed observations for Example 1 are shown in Fig. 3.1 and Fig 3.2 respectively.

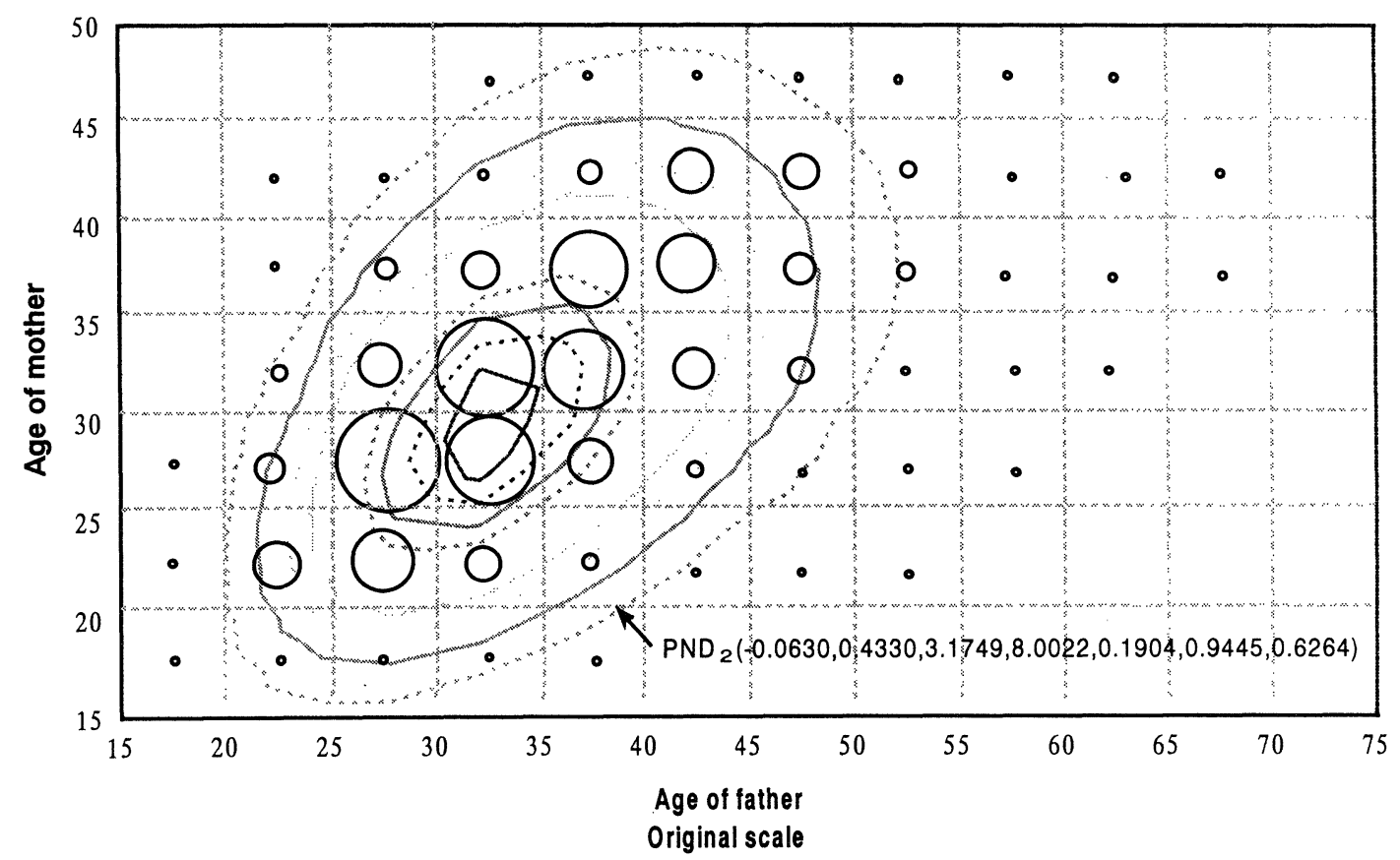

Fig. 3.1 The distributions of the original observations (Example 1) 


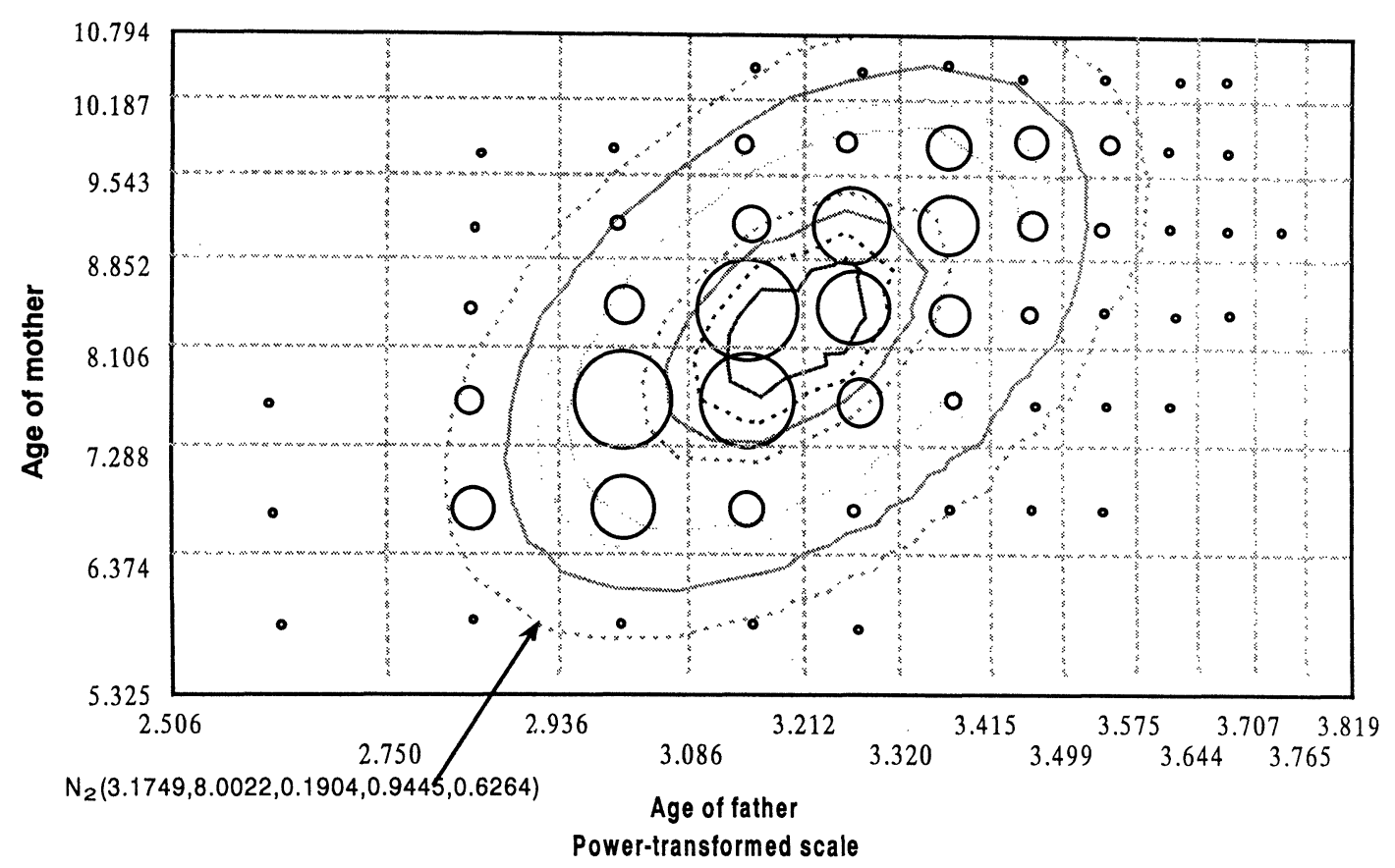

Fig. 3.2 The distributions of the power-transformed observations (Example 1)

\section{An Application of the BPND to Regression}

A bivariate normal regression problem was considered in the framework developed in the previous section. Now our major interest is how to obtain a "good" prediction equation on the basis of observations presented in a correlation table. A simple regression problem will also be mentioned. Haitovsky (1973) have reviewed the problem of regression when observations are grouped. In Fryer and Pethybrige (1972), a generalization of Lindley's methods was proposed to obtain simple estimates of maximum likelihood estimation in the bivariate normal regression model. They considered both of the variables, one variable grouped and the other ungrouped. Furthermore, some departures of the assumptions made by Fryer and Pethybrige (1972) were considered numerically by Pethybrige (1975). These departures are, small samples, non-normality and unequal group widths, which might arise, for instance, when a transformation to normality deemed to be necessary.

\subsection{A Bivariate Regression}

The regression of $Y_{2}$ on $Y_{1}$ is considered. The other case follows by symmetry. If the simultaneous power-transformed variables $\left(Y_{1}^{\left(\lambda_{1}\right)}, Y_{2}^{\left(\lambda_{2}\right)}\right)$ of $\left(Y_{1}, Y_{2}\right)$ have nearly a bivariate normal distribution, for $\lambda_{2} \neq 0$, conditional probability density function of $Y_{2}^{\left(\lambda_{2}\right)}$ given 
$Y_{1}^{\left(\lambda_{1}\right)}=y_{1}^{\left(\lambda_{1}\right)}$ is given by

$$
g\left(y_{2}^{\left(\lambda_{2}\right)} \mid y_{1}^{\left(\lambda_{1}\right)}\right)=\frac{f\left(y_{2}^{\left(\lambda_{2}\right)} \mid y_{1}^{\left(\lambda_{1}\right)}\right)}{\Phi\left\{\frac{\operatorname{sgn}\left(\lambda_{2}\right)}{\sqrt{1-\rho^{2}}}\left(\kappa_{2}+\rho \frac{y_{1}^{\left(\lambda_{1}\right)}-\mu_{1}}{\sigma_{1}}\right)\right\}}
$$

where

$$
f\left(y_{2}^{\left(\lambda_{2}\right)} \mid y_{1}^{\left(\lambda_{1}\right)}\right)=\frac{1}{\sqrt{2 \pi} \sigma_{2} \sqrt{1-\rho^{2}}} \exp \left[-\frac{1}{2 \sigma_{2}^{2}\left(1-\rho^{2}\right)}\left\{y_{2}^{\left(\lambda_{2}\right)}-\mu_{2}-\rho \frac{\sigma_{2}}{\sigma_{1}}\left(y_{1}^{\left(\lambda_{1}\right)}-\mu_{1}\right)\right\}^{2}\right]
$$

Therefore, the conditional expected value of $Y_{2}^{\left(\lambda_{2}\right)}$ given, which for $Y_{1}^{\left(\lambda_{1}\right)}=y_{1}^{\left(\lambda_{1}\right)}$ is given by

$$
\begin{aligned}
\mathrm{E}\left[Y_{2}^{\left(\lambda_{2}\right)} \mid Y_{1}^{\left(\lambda_{2}\right)}=y_{1}^{\lambda_{1}}\right] & =\mu_{2}+\rho \frac{\sigma_{2}}{\sigma_{1}}\left(y_{1}^{\left(\lambda_{2}\right)}-\mu_{1}\right) \\
& +\frac{\sigma_{2} \sqrt{1-\rho^{2}} \phi\left\{\frac{\operatorname{sgn}\left(\lambda_{2}\right)}{\sqrt{1-\rho^{2}}}\left(\kappa_{2}+\rho \frac{y_{1}^{\left(\lambda_{1}\right)}-\mu_{1}}{\sigma_{1}}\right)\right\}}{\Phi\left\{\frac{\operatorname{sgn}\left(\lambda_{2}\right)}{\sqrt{1-\rho^{2}}}\left(\kappa_{2}+\rho \frac{y_{1}^{\left(\lambda_{1}\right)}-\mu_{1}}{\sigma_{1}}\right)\right\}}
\end{aligned}
$$

yields the regression function of $Y_{2}^{\left(\lambda_{2}\right)}$ on $Y_{1}^{\left(\lambda_{1}\right)}$ on the scale of transformed observations. Here is, the regression function of $Y_{2}$ on $Y_{1}$ on the BPND given by

$$
\mathrm{E}\left[Y_{2} \mid Y_{1}=y_{1}\right]=C_{0} \sum_{\nu=0}^{\infty} \frac{(\sqrt{2})^{p+\nu-1}}{\nu !}\left(1-\rho^{2}\right)^{(p-\nu) / 2}\left(\kappa_{2}+\rho \frac{y_{1}^{\left(\lambda_{1}\right)}-\mu_{1}}{\sigma_{1}}\right)^{\nu} \Gamma\left(\frac{p+\nu+1}{2}\right)
$$

where $p=1 / \lambda_{2}$, and

$$
\begin{aligned}
& C_{0}^{\prime}= \\
& \frac{\left(\lambda_{2} \sigma_{2}\right)^{p}}{\sqrt{2 \pi}} \exp \left\{-\frac{1}{2\left(1-\rho^{2}\right)}\left(\kappa_{2}+\rho \frac{y_{1}^{\left(\lambda_{1}\right)}-\mu_{1}}{\sigma_{1}}\right)^{2}\right\}\left[\Phi\left\{\frac{1}{\sqrt{1-\rho^{2}}}\left(\kappa_{2}+\rho \frac{y_{1}^{\left(\lambda_{1}\right)}-\mu_{1}}{\sigma_{1}}\right)\right\}\right]^{-1}
\end{aligned}
$$

where $\Gamma(\cdot)$ denotes the gamma function. As an example, the bivariate regressions on the original scale and the power-transformed scale applied to Example 1 are shown in Fig.4.1 and Fig. 4.2 respectively. 


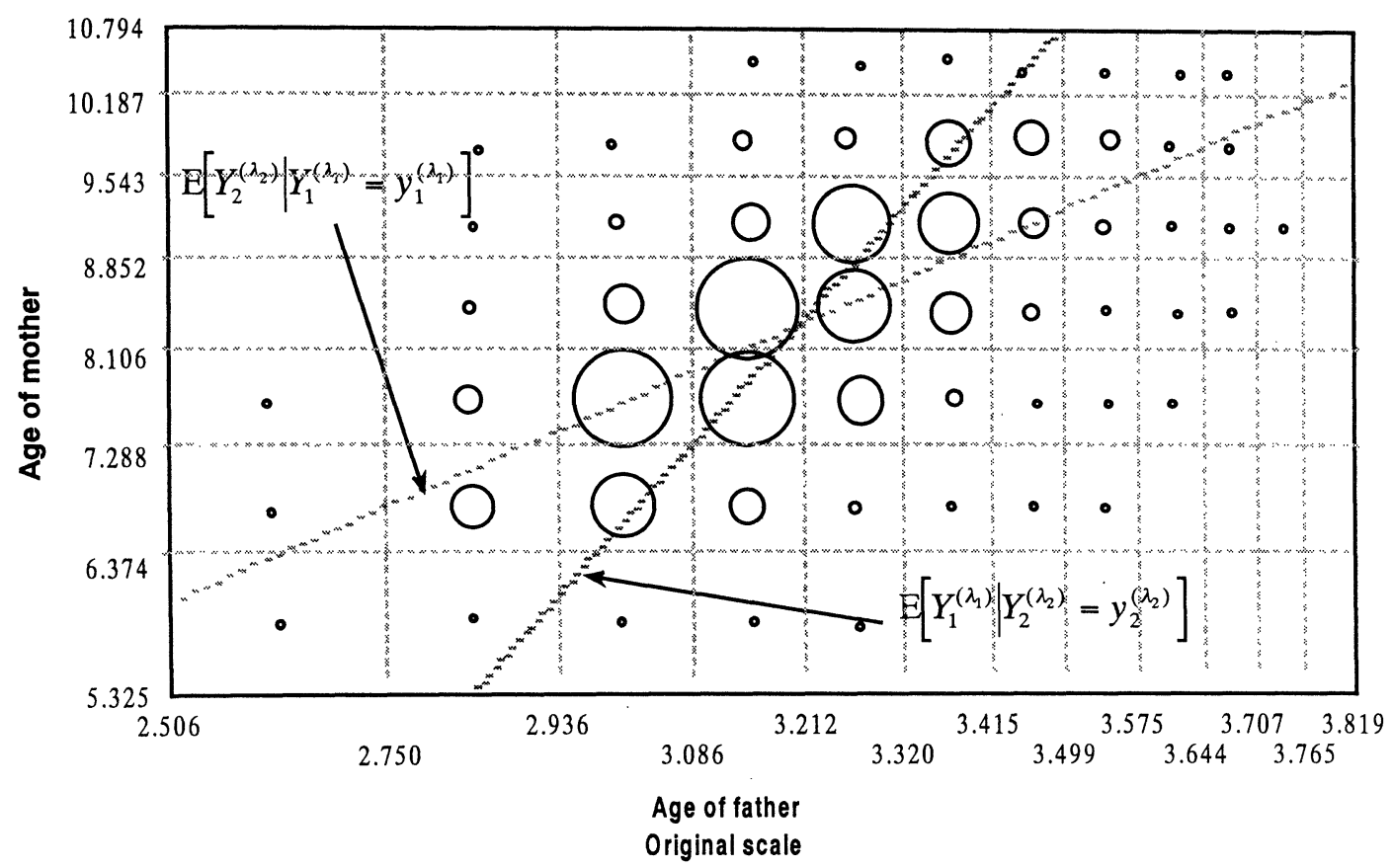

Fig.4.1 The bivariate regressions on the original scale (Example 1)

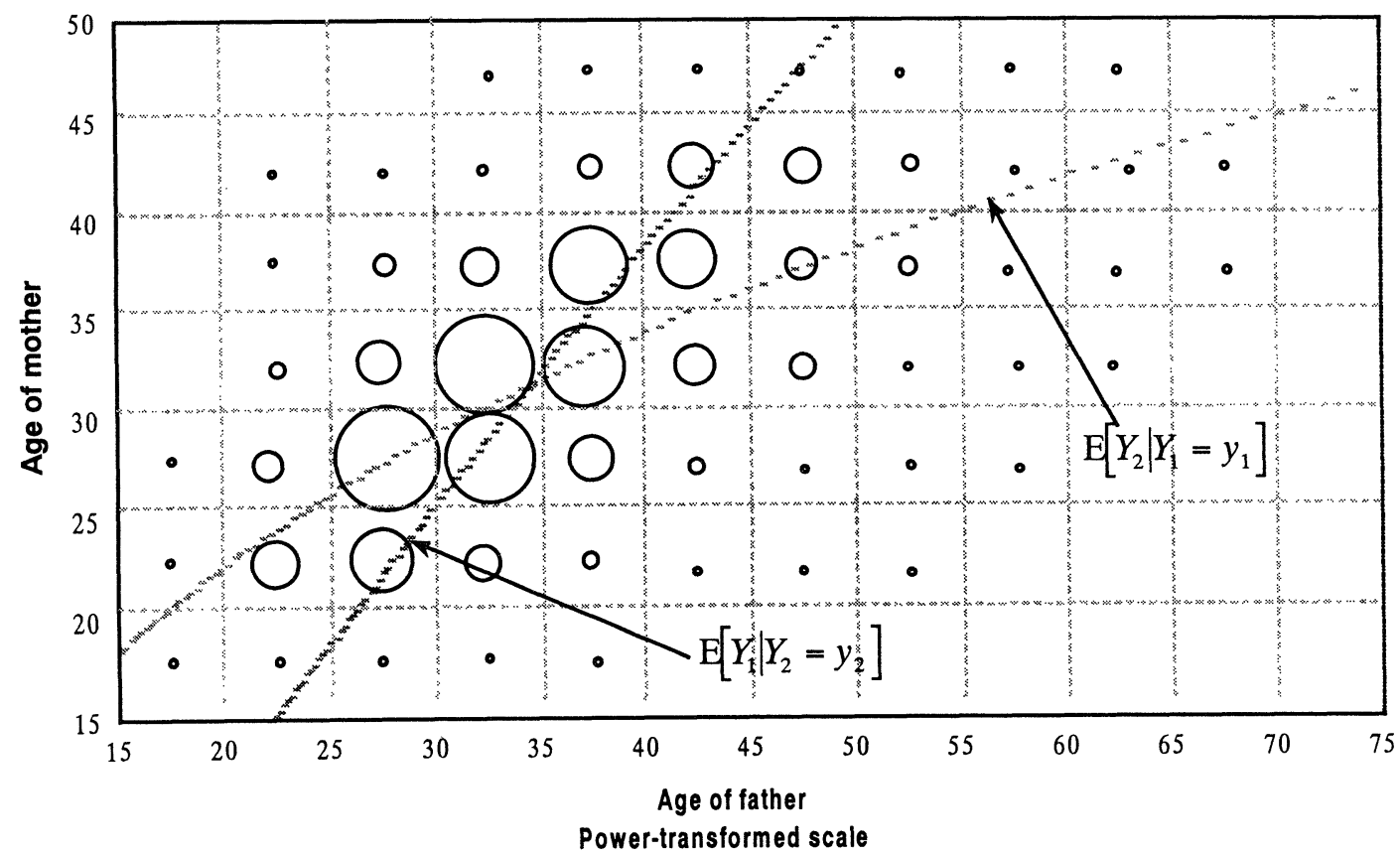

Fig.4.2 The bivariate regressions on the power-transformed scale (Example 1) 


\subsection{A Simple Regression}

In this section, fitting a linear model to a transformed pair of variables $Y^{\left(\lambda_{2}\right)}$ and $x^{\left(\lambda_{1}\right)}$ is investigated, where one or both variables are presented in the grouped form.

\subsubsection{Both Variables in Grouped Form}

In the ordinary regression based on power-transformation, it is assumed that the powertransformed observations $Y^{\left(\lambda_{2}\right)}$ have the linear model. Namely, in the case of simple regression

$$
Y_{u}^{\left(\lambda_{2}\right)}=\beta_{0}+\beta_{1} x_{u}^{\left(\lambda_{1}\right)}+\varepsilon_{u}, \quad u=1, \cdots, n
$$

where $\beta_{0}$ and $\beta_{1}$ are unknown parameters to be estimated, $x_{u}^{\left(\lambda_{1}\right)}$ are known power-transformed independent variables, $\varepsilon_{u}$ are identically and independent distributed $\mathrm{N}\left(0, \sigma^{2}\right)$. The situations mentioned in Section 4.1 are applied to this case.

Suppose that the observations are given in a frequency table like Table 4.2.1, where $x_{0}=y_{0}=0$ and $x_{k}=y_{l}=\infty$. For a given sample size $n$, the quantities $n_{i}$. are supposed to be fixed. In order to carry out calculations with grouped data, it is customary to assume that the observations are placed at the midpoints of the intervals. Namely, for the independent variables, if $x_{u} \in\left[x_{i-1}, x_{i}\right)$, we use the midpoint $x_{M i}^{\left(\lambda_{1}\right)}$ given by

$$
x_{M i}^{\left(\lambda_{1}\right)}=\frac{x_{i-1}^{\left(\lambda_{1}\right)}+x_{i}^{\left(\lambda_{1}\right)}}{2}
$$

in stead of $x_{u}^{\left(\lambda_{1}\right)}$. Therefore, the model (4.2.1) will become

$$
Y_{u}^{\left(\lambda_{1}\right)}=\beta_{0}+\beta_{1} x_{M i}^{\left(\lambda_{1}\right)}+\varepsilon_{i}
$$

for each $u$ such that $x_{u} \in\left[x_{i-1}, x_{i}\right), i=1, \cdots, k$ and $u=1, \cdots, n$. Since $Y_{u}$ is independent

Table 4.2.1. Frequency Table

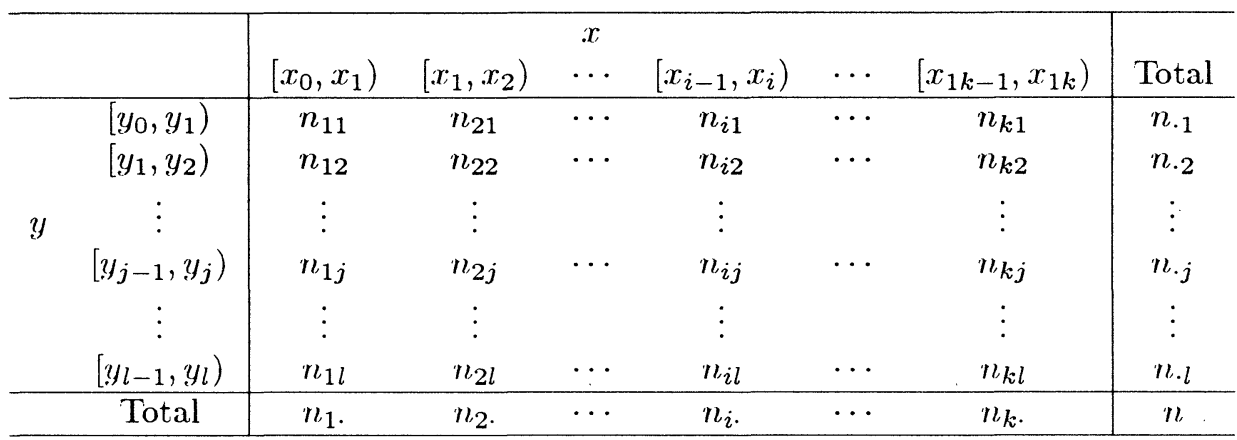

of $Y_{\nu}$ for $u \neq 1$, setting $\boldsymbol{\theta}^{\mathrm{T}}=\left(\lambda_{1}, \lambda_{2}, \beta_{0}, \beta_{1}, \sigma\right)$, the log-likelihood function for the sample of size $n$ is given by

$$
\log L_{n}(\boldsymbol{\theta})=\sum_{i=1}^{k}\left\{\log n_{i} !-\sum_{j=1}^{l} \log n_{i j} !\right\}+\sum_{i=1}^{k} \sum_{j=1}^{l} n_{i j} \log p_{\mathrm{BP} i j}(\boldsymbol{\theta})
$$


where

$$
p_{\mathrm{BP} i j}(\boldsymbol{\theta})=\frac{1}{A(\kappa)}\left\{\Phi\left(\frac{y_{j}^{\left(\lambda_{2}\right)}-\beta_{0}-\beta_{1} x_{i}^{\left(\lambda_{1}\right)}}{\sigma}\right)-\Phi\left(\frac{y_{j-1}^{\left(\lambda_{2}\right)}-\beta_{0}-\beta_{1} x_{i}^{\left(\lambda_{1}\right)}}{\sigma}\right)\right\} .
$$

and

$$
A(\kappa)=\left\{\begin{array}{cl}
\Phi\left(\frac{\lambda_{2}\left(\beta_{0}+\beta_{1} x_{i}^{\left(\lambda_{1}\right)}\right)+1}{\lambda_{2} \sigma}\right), & \lambda_{2}>0 \\
1, & \lambda_{2}=0 \\
\Phi\left(\frac{\lambda_{2}\left(\beta_{0}+\beta_{1} x_{i}^{\left(\lambda_{1}\right)}\right)+1}{\lambda_{2} \sigma}\right), & \lambda_{2}<0 .
\end{array}\right.
$$

\subsubsection{Only One Variables Grouped}

Firstly here, we consider first the case in which the only one dependent variable is grouped. Suppose that $n_{i}$ observations of the variable $Y$ are made at the fixed value $x_{i}$, where $i=1, \cdots, k$ and $\sum_{i=1}^{k} n_{i}$. $=n$ is the total sample size. We deal with this situation exactly as done in the previous case. The only change needed is to substitute the approximate quantities $x_{M i}^{\left(\lambda_{1}\right)}$ used previously, for the exact transformed observations $x_{i}^{\left(\lambda_{1}\right)}$.

The other case is when the outcomes of the random variable $Y$ are exactly specified, but the value of $x$ are grouped into the intervals $\left[0, x_{1}\right),\left[x_{1}, x_{2}\right), \cdots,\left[x_{k-1}, \infty\right)$. In the latter case, we again consider the model (4.2.1) which gives rise to the log-likelihood

$$
\begin{aligned}
\log L_{n}(\boldsymbol{\theta} \mid y) & =-\frac{n}{2} \log 2 \pi-\frac{n}{2} \log \sigma^{2}-\frac{1}{2 \sigma^{2}} \sum_{u=1}^{n} \sum_{i=1}^{k} \delta_{i u}\left(y_{u}^{\left(\lambda_{2}\right)}-\beta_{0}-\beta_{1} x_{M i}^{\left(\lambda_{1}\right)}\right)^{2} \\
& +n\left(\lambda_{1}-1\right) \log y_{u}-n A(\kappa)
\end{aligned}
$$

where

$$
\delta_{i u}=\left\{\begin{array}{lc}
1, & x_{u} \in\left[x_{i-1}, x_{i}\right) \\
0, & \text { otherwise }
\end{array}\right.
$$

\section{Concluding Remarks}

In this paper, in order to investigate the relationship between two variables in which observations of these two variables were grouped into several intervals, the bivariate powernormal distribution were applied to the grouped observations. The results of some examples cited from the published literatures show that the bivariate power-normal distribution will be helpful to "regularize" the observation even when the strict bivariate normality was not achieved. Moreover, a bivariate normal regression and a simple regression were considered in the framework developed in fitting the bivariate power-normal distribution to the grouped observations. Thus, we did not mention the particular case of simple regression, when applying the power-transformation, but there are two difference approaches, i.e., one to achieve the linearity or additivity of the assumed model after the transformation, and another to satisfy the normality of error term or to identify the distribution (on the untransformed scale) before the transformation, namely the PND. It is suitable that the power-transformation is applied on the basis of these approaches (Goto et al., 1983: Goto et al., 1987: Goto et al., 1991: Goto, 1992. 1995). 


\section{Acknowledgements}

The authors would like to thank the editor and two referees for their helpful comments and suggestions on this paper.

\section{APPENDIX 1 : The Asymptotic Properties of the Estimators on the BPND}

In this section, the asymptotic properties of the maximum likelihood estimators obtained in fitting of the BPND are considered by using the approach to discrete distributions by Hernandez and Johnson (1981).

Let $\boldsymbol{\theta}^{\mathrm{T}}=\left(\theta_{1}, \theta_{2}, \theta_{3}, \theta_{4}, \theta_{5}, \theta_{6}, \theta_{7}\right)=\left(\lambda_{1}, \lambda_{2}, \mu_{1}, \mu_{2}, \sigma_{1}, \sigma_{2}, \rho\right), \hat{p}_{i j, n}=n_{i j} / n$, and

$$
p_{i j}=\int_{y_{1 i-1}}^{y_{1 i}} \int_{y_{2 j-1}}^{y_{2 j}} g^{*}\left(y_{1}, y_{2}\right) d y_{1} d y_{2}
$$

where $g^{*}$ is the true probability density function. Suppose that the parameter space $\Theta$ is a compact set given by $\Theta=\left\{\boldsymbol{\theta}=\left(\lambda_{1}, \lambda_{2}, \mu_{1}, \mu_{2}, \sigma_{1}, \sigma_{2}, \rho\right)^{\mathrm{T}}\right\}$ and that

$$
H(\boldsymbol{\theta})=\sum_{i=1}^{k} p_{i j} \log \left\{p_{\mathrm{BP} i j}(\boldsymbol{\theta}) / p_{i j}\right\}
$$

which has a unique global maximum at $\boldsymbol{\theta}_{0} \in \Theta$ is a continuos function. Also, let $\boldsymbol{\theta}_{0}$ be an interior point of $\Theta$, and Hessian $\nabla^{2} H\left(\boldsymbol{\theta}_{0}\right)$ of $H(\boldsymbol{\theta})$ be nonsingular at $\boldsymbol{\theta}_{0}$.

Stirling's approximation yields

$$
\begin{aligned}
\frac{1}{n} \log n !-\frac{1}{n} \sum_{i=1}^{k} \sum_{j=1}^{l} \log n_{i j} ! & =\frac{1-k l}{2 n} \log 2 \pi-\sum_{i=1}^{k} \sum_{j=1}^{l} \hat{p}_{i j, n} \log \hat{p}_{i j, n} \\
& +\frac{1}{2 n}\left\{(1-k l) \log n-\sum_{i=1}^{k} \sum_{j=1}^{l} \log \hat{p}_{i j, n}\right\}+O_{p}\left(n^{-1}\right)(A
\end{aligned}
$$

By (A1.1)

$$
\frac{1}{n} \log L_{n}(\boldsymbol{\theta})=\sum_{i=1}^{k} \sum_{j=1}^{l} \hat{p}_{i j, n} \log p_{\mathrm{BP} i j}(\boldsymbol{\theta}) \sum_{i=1}^{k} \sum_{j=1}^{l} \hat{p}_{i j, n} \log \hat{p}_{i j, n}(\boldsymbol{\theta})+o_{p}(1) .
$$

Therefore, the inequality

$$
\begin{aligned}
& \left|\frac{1}{n} \log L_{n}(\boldsymbol{\theta})-\sum_{i=1}^{k} \sum_{j=1}^{l} p_{i j} \log \frac{p_{\mathrm{BP} i j}(\boldsymbol{\theta})}{p_{i j}}\right| \\
& \leq\left|\sum_{i=1}^{k} \sum_{j=1}^{l} \hat{p}_{i j, n} \log p_{\mathrm{BP} i j}(\boldsymbol{\theta}) \sum_{i=1}^{k} \sum_{j=1}^{l} p_{i j} \log p_{\mathrm{BP} i j}(\boldsymbol{\theta})\right| \\
& \quad+\left|\sum_{i=1}^{k} \sum_{j=1}^{l} \hat{p}_{i j, n} \log \hat{p}_{i j, n} \sum_{i=1}^{k} \sum_{j=1}^{l} p_{i j} \log \hat{p}_{i j}\right|+o_{p}(1)
\end{aligned}
$$


hold. Thus, because it follows from Appendix 2 that as $n \rightarrow \infty$ and $\hat{p}_{i j, n}=n_{i j} / n \rightarrow$ const., for $\boldsymbol{\theta} \in \Theta$

$$
\left|\sum_{i=1}^{k} \sum_{j=1}^{l} \hat{p}_{i j, n} \log p_{\mathrm{BP} i j}(\boldsymbol{\theta})-\sum_{i=1}^{k} \sum_{j=1}^{l} p_{i j} \log p_{\mathrm{BP} i j}(\boldsymbol{\theta})\right| \stackrel{a . s .}{\longrightarrow} 0
$$

and continuity of $x \log x$, and $\hat{p}_{i j, n} \stackrel{\text { a.s. }}{\longrightarrow} p_{i j}$ as $n \rightarrow \infty$, the right hand side of inequality (A1.3) goes to zero with probability one uniformly in $\boldsymbol{\theta} \in \Theta$. Hence, as $n \rightarrow \infty$, for $\boldsymbol{\theta} \in \Theta$

$$
\frac{1}{n} \log L_{n}(\boldsymbol{\theta}) \stackrel{\text { a.s. }}{\longrightarrow} H(\boldsymbol{\theta}) \text {. }
$$

Therefore, it follows from Appendix 3 that $\hat{\boldsymbol{\theta}}_{n} \stackrel{\text { a.s }}{\longrightarrow} \boldsymbol{\theta}_{0}$ as $n \rightarrow \infty$ form by identifying $H(\boldsymbol{\theta})$ with the function $f(\boldsymbol{\theta})$ of the Appendix.

In order to show the asymptotic normality of $\boldsymbol{\theta}_{n}$, the gradient and the Hessian of loglikelihood function are considered. The gradient of $\log L_{n}(\boldsymbol{\theta})$ is given by

$$
\nabla \log L_{n}(\boldsymbol{\theta})=\left(\frac{\partial \log L_{n}(\boldsymbol{\theta})}{\partial \theta_{u}}\right), \quad u=1, \cdots, 7
$$

and the Hessian of $\log L_{n}(\boldsymbol{\theta})$ is the $7 \times 7$ symmetric matrix $\nabla^{2} \log L_{n}(\boldsymbol{\theta})=\left(h_{u v, n}(\boldsymbol{\theta})\right)$ with its elements

$$
h_{u v, n}(\boldsymbol{\theta})=\frac{\partial^{2} \log L_{n}(\boldsymbol{\theta})}{\partial \theta_{u} \partial \theta_{v}}, \quad u, v=1, \cdots, 7
$$

It is readily seen that the second partial derivatives are continuous on $\Theta$. Using Taylor's formula to expand $n^{-1 / 2} \nabla \log L_{n}\left(\hat{\boldsymbol{\theta}}_{n}\right)$ about $\boldsymbol{\theta}_{0}$

$$
\frac{1}{\sqrt{n}} \nabla \log L_{n}\left(\hat{\boldsymbol{\theta}}_{n}\right)=\frac{1}{\sqrt{n}} \nabla \log L_{n}\left(\boldsymbol{\theta}_{0}\right)+\frac{1}{n} \nabla^{2} \log L_{n}\left(\boldsymbol{\theta}_{n}^{*}\right)\left\{\sqrt{n}\left(\hat{\boldsymbol{\theta}}_{n}-\boldsymbol{\theta}_{0}\right)\right\}
$$

is obtained, where $\boldsymbol{\theta}_{n}^{*}=\gamma_{n} \boldsymbol{\theta}_{0}+\left(1-\gamma_{n}\right) \hat{\boldsymbol{\theta}}_{n} \quad\left(0<\gamma_{n}<1\right)$.

Next since $\hat{\boldsymbol{\theta}}_{n} \stackrel{a . s}{\longrightarrow} \boldsymbol{\theta}_{0}$ as $n \rightarrow \infty$, with the assumption that $\boldsymbol{\theta}_{0}$ is an interior point of $\Theta$, $\nabla \log L_{n}(\hat{\boldsymbol{\theta}})=\mathbf{o}$ for all sufficiently large, on an almost sure set. Therefore, as $n \rightarrow \infty$

$$
\frac{1}{\sqrt{n}} \nabla \log L_{n}\left(\boldsymbol{\theta}_{0}\right) \frac{1}{n} \nabla^{2} \log L_{n}\left(\boldsymbol{\theta}_{n}^{*}\right)\left\{\sqrt{n}\left(\hat{\boldsymbol{\theta}}-\boldsymbol{\theta}_{0}\right)\right\} \stackrel{\text { a.s. }}{\longrightarrow} \mathbf{o}
$$

so that $n^{-1 / 2} \nabla \log L_{n}\left(\boldsymbol{\theta}_{0}\right)$ and $-n^{-1} \nabla^{2} \log L_{n}\left(\boldsymbol{\theta}_{n}^{*}\right)\left\{\sqrt{n}\left(\hat{\boldsymbol{\theta}}_{n}-\boldsymbol{\theta}_{0}\right)\right\}$ have the same limiting distri bution.

Since we can write

$$
\frac{1}{\sqrt{n}} \nabla \log L_{n}\left(\boldsymbol{\theta}_{0}\right)=\frac{1}{n} \sum_{m=1}^{n}\left[\sum_{i=1}^{k} \sum_{j=1}^{l} D_{I_{i j}}\left(y_{m}\right) \alpha_{i j}\left(\boldsymbol{\theta}_{0}\right)\right]=\frac{1}{n} \sum_{m=1}^{n} \boldsymbol{X}_{m}\left(\boldsymbol{\theta}_{0}\right)
$$

where $D_{i j}$ is the indicator function of the set $I_{i j}$, that is, $D_{I_{i j}}\left(y_{m}\right)=1$ if $y_{m} \in I_{i j}$ and $D_{I_{i j}}\left(y_{m}\right)=0$ if $y_{m} \notin I_{i j}, \alpha_{i j}\left(\boldsymbol{\theta}_{0}\right)=\left(\alpha_{i j r}\left(\boldsymbol{\theta}_{0}\right)\right)$ and

$$
\alpha_{i j r}\left(\boldsymbol{\theta}_{0}\right)=\left(\left.\frac{\partial \log p_{\mathrm{BP} i j}(\boldsymbol{\theta})}{\partial \theta_{r}}\right|_{\theta=\theta_{0}}\right), \quad r=1, \cdots, 7
$$


then the random vectors $\boldsymbol{X}_{1}\left(\boldsymbol{\theta}_{0}\right), \cdots, \boldsymbol{X}_{n}\left(\boldsymbol{\theta}_{0}\right)$ are independent and identically distributed with $\mathrm{E}\left[\boldsymbol{X}_{m}\left(\boldsymbol{\theta}_{0}\right)\right]=\nabla H\left(\boldsymbol{\theta}_{0}\right)=\mathbf{o}$ and $\mathrm{E}\left[\boldsymbol{X}_{m}\left(\boldsymbol{\theta}_{0}\right) \boldsymbol{X}_{m}^{\mathrm{T}}\left(\boldsymbol{\theta}_{0}\right)\right]=\boldsymbol{W}$, where $\boldsymbol{W}=\left(w_{u v}\right)_{7 \times 7}$ is given by

$$
\begin{aligned}
w_{u v} & =\sum_{i=1}^{k} \sum_{i=1}^{l} p_{i j} \alpha_{i j u}\left(\boldsymbol{\theta}_{0}\right) \alpha_{i j v}\left(\boldsymbol{\theta}_{0}\right) \\
& =\sum_{i=1}^{k} \sum_{i=1}^{l} p_{i j}\left(\left.\frac{\partial \log p_{\mathrm{BP} i j}(\boldsymbol{\theta})}{\partial \theta_{u}}\right|_{\theta=\theta_{0}}\right)\left(\left.\frac{\partial \log p_{\mathrm{BP} i j}(\boldsymbol{\theta})}{\partial \theta_{v}}\right|_{\theta=\theta_{0}}\right) .
\end{aligned}
$$

Thus, an application of the multivariate central limit theorem yields, as $n \rightarrow \infty$

$$
\frac{1}{\sqrt{n}} \log L_{n}\left(\boldsymbol{\theta}_{0}\right) \stackrel{d}{\longrightarrow} \mathrm{N}_{7}(\mathbf{o}, \boldsymbol{W}) .
$$

Furthermore, we can write

$$
\frac{1}{n} h_{u v, n}(\boldsymbol{\theta})=\sum_{i=1}^{k} \sum_{i=1}^{l} \hat{p}_{i j, n} \delta_{i j, u v}(\boldsymbol{\theta})
$$

where $\delta_{i j, u v}(\boldsymbol{\theta})$ is uniformly continuous for $\boldsymbol{\theta} \in \Theta$ and for each $u$ and $v$. So, the fact that $\hat{p}_{i j, n} \stackrel{a . s .}{\longrightarrow} p_{i j}$ as $n \rightarrow \infty$ implies that uniformly on $\Theta$

$$
\frac{1}{n} h_{i j, n}(\boldsymbol{\theta}) \stackrel{a . s .}{\longrightarrow} \frac{\partial^{2}}{\partial_{u} \partial_{v}}\left(\sum_{i=1}^{k} \sum_{j=1}^{l} p_{i j} \log \frac{p_{\mathrm{BP} i j}(\boldsymbol{\theta})}{p_{i j}}\right)=\frac{\partial^{2} H(\boldsymbol{\theta})}{\partial \theta_{u} \partial \theta_{v}}
$$

Hence, as $\boldsymbol{\theta}_{n}^{*}$ lies on the segment jointing $\hat{\boldsymbol{\theta}}_{n}$ and $\boldsymbol{\theta}_{0}$, we have as $n \rightarrow \infty$

$$
\left.\frac{1}{n} h_{i j, n}\left(\boldsymbol{\theta}_{n}^{*}\right) \stackrel{p}{\longrightarrow} \frac{\partial^{2} H(\boldsymbol{\theta})}{\partial \theta_{u} \partial \theta_{v}}\right|_{\theta=\theta_{0}} .
$$

Therefore, by setting $\boldsymbol{V}=\left\{\nabla^{2} H\left(\boldsymbol{\theta}_{0}\right)\right\}^{-1}$, Slutsky's theorem can be applied to finally conclude that as $n \rightarrow 0$

$$
\sqrt{n}\left(\hat{\boldsymbol{\theta}_{n}}-\boldsymbol{\theta}_{0}\right) \stackrel{\text { a.s. }}{\longrightarrow} \mathrm{N}_{7}\left(\mathbf{o}, \boldsymbol{V} \boldsymbol{W} \boldsymbol{V}^{\mathrm{T}}\right)
$$

These results show that strong consistency and asymptotic normality of the maximum likelihood estimators of $\lambda_{1}, \lambda_{2}, \mu_{1}, \mu_{2}, \sigma_{1}, \sigma_{2}$, and $\rho$.

\section{APPENDIX 2}

Assume that the parameter space $\Theta$ is a compact subset of $R^{7}$. The maximum likelihood estimators of $\boldsymbol{\theta}=\left(\lambda_{1}, \lambda_{2}, \mu_{1}, \mu_{2}, \sigma_{1}, \sigma_{2}, \rho\right)^{\mathrm{T}}$ will be defined as the value of $\boldsymbol{\theta} \in \Theta$ which, for a given sample of size $n$, gives a global maximum for $\log L_{n}(\boldsymbol{\theta})$. The value is denoted by $\hat{\boldsymbol{\theta}}_{n}$.

Let $i j$ th cell $I_{i j}, p_{i j}=\iint_{I_{i j}} g^{*}\left(y_{1}, y_{2}\right) d y_{1} d y_{2}, \hat{p}_{i j, n}=n_{i j} / n, p_{\mathrm{BP} i j}(\boldsymbol{\theta})$ be as in $(2.2 .2)$, where $g^{*}$ is the true probability density function. And $D_{I}(\cdot)$ be the indicator function of the set $I$, that is

$$
D_{I}(y)=\left\{\begin{array}{lc}
1, & y \in I \\
0, & \text { otherwise. }
\end{array}\right.
$$




\section{HAMASAKI and GOTO}

For each $i=1, \cdots, k, j=1, \cdots, l$ consider the sequence of independent and identically distributed 0-1 random variables $\left\{D_{I_{i j}\left(y_{m}\right)}, m=1, \cdots, n\right\}$ with success probabilities $\mathrm{P}_{g^{*}}\left[D_{I_{i j}}\left(y_{m}\right)=\right.$ 1] $=p_{i j}$, where $\mathrm{P}_{g^{*}}(\cdot)$ is written to emphasize that the probability is determined with respect to the true probability density function $g^{*}$.

Since $n_{i j}=\sum_{m=1}^{n} D_{I_{i j}}\left(y_{m}\right)$, it follows from the strong law of large numbers that as $n \rightarrow \infty$

$$
\hat{p}_{i j, n} \stackrel{a . s .}{\longrightarrow} p_{i j} .
$$

Let $A=\left\{\omega \in \Theta \mid \hat{p}_{i j, n}(\omega) \rightarrow p_{i j}\right.$ as $\left.n \rightarrow \infty\right\}$. Then, for $\varepsilon>0$ and $\omega \in A$, we obtain,

$$
\begin{aligned}
\left|\sum_{i=1}^{k} \sum_{j=1}^{l}\left(\hat{p}_{i j, n}(\omega)-p_{i j}\right) \log p_{\mathrm{BP} i j}\right| & \leq \sum_{i=1}^{k} \sum_{j=1}^{l}\left|\hat{p}_{i j, n}(\omega)-p_{i j}\right|\left|\log p_{\mathrm{BP} i j}(\boldsymbol{\theta})\right| \\
& \leq \varepsilon\left|\log p_{\mathrm{BP} i j}(\boldsymbol{\theta})\right|
\end{aligned}
$$

for ${ }^{\forall} n \geq \mathrm{N}(\varepsilon, \omega)$ and $\boldsymbol{\theta} \in \Theta$.

Next, since $Y_{1 i}^{\lambda_{1}}$ and $Y_{2 i}^{\lambda_{2}}$ are monotone increasing in $\lambda_{1}$ and $\lambda_{2}$ respectively, and $Y_{1 i-1}<$ $Y_{1 i}$ and $Y_{2 j-1}<Y_{2 j}$ for each $i=1, \cdots, k, j=1, \cdots, l$, it follows easily that $p_{\mathrm{BP} i j}(\boldsymbol{\theta})>0$ on $\Theta$. Also, from the continuity of $z_{1 i}(\boldsymbol{\theta})=\left(y_{1 i}^{\left(\lambda_{1}\right)}-\mu_{1}\right) / \sigma_{1}$ and $z_{2 i}(\boldsymbol{\theta})=\left(y_{2 i}^{\left(\lambda_{2}\right)}-\mu_{2}\right) / \sigma_{2}$ for $\boldsymbol{\theta} \in \Theta$, we have it that $\log p_{\mathrm{BP} i j}$ is continuous on $\Theta$. This result and $\Theta$ being compact by assumption imply the existence of $R_{i j}=\inf _{\theta \in \Theta} \log p_{\mathrm{BP} i j}(\boldsymbol{\theta})>-\infty$. Hence for any $\boldsymbol{\theta} \in \Theta$

$$
\sum_{i=1}^{k} \sum_{j=1}^{l}\left|\log p_{\mathrm{BP} i j}(\boldsymbol{\theta}) \leq R\right|
$$

where $R=\sum_{i=1}^{k} \sum_{j=1}^{l}\left|R_{i j}\right|<\infty$. Therefore, it follows from (A1.2) and (A1.3) that as $n \rightarrow \infty$

$$
\left|\sum_{i=1}^{k} \sum_{j=1}^{l} \hat{p}_{i j, n} \log p_{\mathrm{BP} i j}(\boldsymbol{\theta})-\sum_{i=1}^{k} \sum_{j=1}^{l} p_{i j} \log p_{\mathrm{BP} i j}(\boldsymbol{\theta})\right| \stackrel{\text { a.s. }}{\longrightarrow} 0 .
$$

\section{APPENDIX 3}

Assume that the parameter space $\Theta$ is a compact subset of $R^{7}$. The maximum likelihood estimators of $\boldsymbol{\theta}=\left(\lambda_{1}, \lambda_{2}, \mu_{1}, \mu_{2}, \sigma_{1}, \sigma_{2}, \rho\right)^{\mathrm{T}}$ will be defined as the value of $\boldsymbol{\theta} \in \Theta$ which, for a given sample of size $n$, gives a global maximum for $\log L_{n}(\theta)$ given in (2.2.4). The value is denoted by $\hat{\boldsymbol{\theta}}_{n}$. Also, suppose that $f$ is a continuous function drown from $\Theta$ to $R$, $n^{-1} \log L_{n}(\boldsymbol{\theta}) \rightarrow \int(\boldsymbol{\theta})$ uniformly in $\boldsymbol{\theta} \in \Theta, f(\boldsymbol{\theta})$ has a unique global maximum at $\boldsymbol{\theta}=\boldsymbol{\theta}_{0}$.

To obtain a contradiction, suppose that $\hat{\boldsymbol{\theta}}_{n}$ does not converge almost surely to $\boldsymbol{\theta}_{0}$. That is, assume there exists a set $B$ such that for each $\omega \in B, \hat{\boldsymbol{\theta}}_{n}(\omega) \neq \boldsymbol{\theta}_{0}$ as $n \rightarrow 0$, where $\mathrm{P}(I)>0$. Let $C^{\prime}=\left\{\omega \mid n^{-1} \log L_{n}(\boldsymbol{\theta}) \rightarrow f(\boldsymbol{\theta})\right.$ as $n \rightarrow \infty$, uniformly in $\left.\boldsymbol{\theta} \in \Theta\right\}$ be and consider $I)=B \cap(;, \mathrm{P}(I))=\mathrm{P}(B)>0$.

Since $\Theta$ is compact for each $\omega \in D$, there exists a subsequence and the limit point $\{m\} \subset\{n\}$ depending on $\omega$ such that $\hat{\boldsymbol{\theta}}(\omega) \rightarrow \boldsymbol{\theta}_{\omega}$ as $m \rightarrow \infty$, with $\boldsymbol{\theta}_{\omega}=\boldsymbol{\theta}_{0}$. For a fixed $\omega \in D$, we have

$$
\left|\frac{1}{m} \log L_{m}\left(\hat{\boldsymbol{\theta}}_{m}(\omega)\right)-\int\left(\boldsymbol{\theta}_{\omega}\right)\right|
$$




$$
\begin{aligned}
& \leq\left|\frac{1}{m} \log L_{m}\left(\hat{\boldsymbol{\theta}}_{m}(\omega)\right)-f\left(\boldsymbol{\theta}_{m}(\omega)\right)\right|+\left|f\left(\hat{\boldsymbol{\theta}}_{m}(\omega)\right)-f\left(\boldsymbol{\theta}_{\omega}\right)\right| \\
& \leq \max _{\theta \in \Theta}\left|\frac{1}{m} \log L_{m}(\boldsymbol{\theta}(\omega))-f(\boldsymbol{\theta}(\omega))\right|+\left|f\left(\hat{\boldsymbol{\theta}}_{m}(\omega)\right)-f\left(\boldsymbol{\theta}_{\omega}\right)\right| .
\end{aligned}
$$

Then, letting $m \rightarrow \infty$ be on the right hand side of (A2.1), we get

$$
\max _{\theta \in \Theta}\left|\frac{1}{m} \log L_{m}(\boldsymbol{\theta}(\omega))-f(\boldsymbol{\theta}(\omega))\right| \rightarrow 0
$$

because $D=B \cap C$ and $\left|f\left(\hat{\boldsymbol{\theta}}_{m}(\omega)\right)-f\left(\boldsymbol{\theta}_{\omega}\right)\right| \rightarrow 0$ by the continuity of $f$. Thus, for $\omega \in D$, as $n \rightarrow \infty$

$$
\frac{1}{m} \log L_{m}\left(\hat{\boldsymbol{\theta}}_{m}(\omega)\right) \rightarrow f\left(\boldsymbol{\theta}_{0}\right)
$$

However, by definition of $\hat{\boldsymbol{\theta}}_{m}$, we have

$$
\frac{1}{m} \log L_{m}\left(\hat{\boldsymbol{\theta}}_{m}\right) \geq \frac{1}{m} \log L_{m}\left(\hat{\boldsymbol{\theta}}_{0}\right)
$$

so that, taking the limit of (A3.4), we obtain $f\left(\boldsymbol{\theta}_{\omega}\right) \geq f\left(\boldsymbol{\theta}_{\omega}\right)$ for $\omega \in D$. Since $\mathrm{P}(D)>0$ contradicts the assumption that $\boldsymbol{\theta}_{0}$ gives a unique maximum and we conclude that $\hat{\boldsymbol{\theta}}_{\boldsymbol{n}} \stackrel{\boldsymbol{a} \cdot \boldsymbol{s} \text {. }}{\longrightarrow} \boldsymbol{\theta}_{0}$ as $n \rightarrow \infty$ as asserted.

\section{REFERENCES}

Atkinson, A.C., Perocchi, L.R. and Smith, R.L. (1991). Grouped likelihood for shifted power transformation. J. Roy. Statist. Soc., B53, 473-482.

Barnard, G.A. (1967). The use of the likelihood function in statistical practice. The 5th Berkley Symp. Mathematical Statistics \& Probability, ed. by L.M. LeCam \& J. Neyman, 1, 27-40.

Borkowf, C.B., Gail, M.H., Carroll, R.J. and Gill, R.D. (1997). Analyzing bivariate continuous data grouped into categories defined by empirical quantiles of marginal distributions. Biometrics, 53, 1054-1069.

Box, G.P.E. and Cox, D.R. (1964). An analysis of transformations (with discussion). J. Roy. Statist. Soc., B26, 211-252.

Copas, J.B. (1972). The likelihood surface in the linear functional relationship. J. Roy. Statist. Soc., B34, 274-278.

Cox, D.R. (1957). Note on grouping. J. Amer. Statist. Assoc., 52, 543-547.

Cramér, H. (1974). Mathematical Methods of Statistics. Princeton University Press.

Dahiya, R.C. and Gurland, J.(1973). A test of fit for bivariate distributions. J. Roy. Statist. Soc., B35, 452-465.

Domae, A. and Miyahara, H. (1984). Are the cubic roots of body weights distributed normally?: Processing of health data and fitting of them to a type of distribution by means of microcomputer(in Japanese). Jpn. J. Behav., 11(2), 1-11.

Fryer, J.G. and Pethybeigde, R.J. (1972). Maximum likelihood estimation of a linear regression function with grouped data. Appl. Statist., 21, 142-154.

Giesbrecht, F. and Kempthorne, O. (1976). Maximum likelihood estimation in three-parameter log-normal distribution. J. Roy. Statist. Soc., B38, 257-264.

Goto, M. (1986). Process of statistical data analysis (in Japanese). Jpn. J. Behav., 13(2), 48-63. 
Goto, M. (1992). Extensive views of power transformations: some recent developments. Invited paper of Honolulu Conference on Computational Statistics as Memorial of the 5th Anniversary JSCS, JAMS, December, 1-5.

Goto, M. (1995). Double power-transformations and their applications. Invited paper of International Conference on Statistical Computing for Quality and Productivity Improvement, Seoul, August, 17-19.

Goto, M., Inoue, T. and Nagai, T. (1980). A bivariate power-normal distribution (in Japanese). Proceedings of the Autumn Meeting of Mathematical Society of Japan, 77-78.

Goto, M., Inoue, T. and Nagai, T. (1981a). A bivariate power-normal distribution (in Japanese). Proceedings of the Spring Meeting of Mathematical Society of Japan, 97-98.

Goto, M., Inoue, T. and Nagai, T. (1981b). A bivariate power-normal distribution (in Japanese). Proceedings of the Autumn Meeting of Mathematical Society of Japan, 63-64.

Goto, M., Inoue, T. and Nagai, T. (1982). The bivariate power normal distributions and its applications. Proceedings of the 1st Korea and Japan Joint Conference of Statistics, Okayama, July $16-17$.

Goto, M., Inoue, T. and Tsuchiya, Y. (1984). On estimation of parameters in power-normal distribution. Bull. Inform. Cyber., 21(1-2), 41-53.

Goto, M., Inoue, T. and Tsuchiya, Y. (1987). Double power-transformation and its performance: An extensive version of Box-Cox transformation. J. Japan. Statist. Soc., 17(2), 149-163.

Goto, M., Matsubara, Y. and Tsuchiya, Y. (1983). Power-normal distribution and its applications. Rep. Stat. Appl. Res., JUSE., 30(3), 8-28.

Goto, M., Yamamoto, Y. and Inoue. T. (1991). Parameter estimation for power-normal distribution: Asymptotic behavior of the estimators(in Japanese). Bull. Comput. Statist. Jpn., 4(1), 45-60.

Haitovsky, Y. (1973). Regression Estimation from Grouped Observations. Griffin.

Hamasaki, T. and Goto, M. (1997). Applications of power-normal distribution to grouped observations. Proceedings of the 9th Korea and Japan Joint Conference of Statistics, Cheju, December 5-6.

Hamasaki, T. and Goto, M. (1998). Fitting of power-normal distribution to grouped observations (in Japanese). Jpn.J.Behav., 25(1), 10-18.

Hernandez, F. and Johnson, R.A. (1981). Transformation of a discrete distribution to near normality. Statistical Distributions in Scientific Work, 5, 259-270.

Holmes, J. (1974). The relative burden of higher gasoline prices. In Five Thousand American Families: Patterns of Economic Progress. Vol. 4, Ann Arbor, Institute for Social Research.

Jimura, Y., Kawai, N. and Goto, M. (1996). Bivariate power-normal distribution and its evaluations (in Japanese). Proceedings of the 13th Oita Meeting about Statistical Science.

Kariya, T. (1975). Menarche age distribution estimated by the methods of maximum likelihood on incomplete quantal response data(in Japanese). Kawasaki Medical J., 1, 75-83.

Kariya, T. (1979). The eruption age distribution of deciduous teeth (in Japanese). Jpn. J. Appl. Statist., 9(2), 73-81.

Kariya, T. and Akasaka, M(1976). The eruption age distribution of deciduous teeth: Maximum likelihood estimation of parameters based on incomplete quantal response data (in Japanese). Jpn. J. Appl. Statist., 5(1), 3-18.

Kawai, N., Jimura, Y. and Goto, M. (1996). Bivariate power-normal distribution and its applications (in Japanese). Proceedings of 10th Conference of Japanese Society of Computational Statistics, 96-99.

Kempthorne, O. (1966). Some aspects of experimental inference. J. Amer. Statist. Assoc., 61, 11-34.

Kempthorne, O. and Folks, L. (1971). Probability, Statistics, and Data Analysis. Iowa University Press. 
Kendall, M.G. and Buckland, W.R. (1982). A Dictionary of Statistical Terms. 4th Edition, The Longman Group[Statistics Group of Chiba University (1987). Kendall toukeigaku yougo jiten. Maruzen].

Kitagawa, T and Inaba, M.(1979). Toukeigaku-tsuron (in Japanese). 2nd edition, Kyoritsu.

Kulldorff, G. (1962). Contributions to the Theory of Estimation From Grouped and Partially Grouped Samples. Almqvist \& Wiksell.

Mardia, K.V. (1970). Families of Bivariate Distribution. Griffin.

Matsubara, Y. and Goto, M. (1979). A censored regression analysis of survival time data based on power-normal distribution (in Japanese). Jpn. J. Appl. Statist., 9(1), 43-51.

Nabeya, S. (1983). On maximum likelihood for grouped observations (in Japanese). Jpn. J. Appl. Statist., 12(2), 59-67.

Pethybridge, R.J. (1975). Maximum likelihood estimation of linear regression function with grouped data. Appl. Statist., 24, 28-41.

Shibata, Y. (1981). Seiki bunpu (in Japanese). University of Tokyo Press.

Stuart, A. and Ord, J.K. (1986). Kendall's Advanced Theory of Statistics. Vol.1, 6th edition, Edward Arnold.

Sugiura, N. (1980). Fitting of some distributions to total score of the common first-stage examination for university entrance (in Japanese). Jpn. J. Appl. Statist., 9(2), 95-107.

Sugiura, N. (1981). Fitting of some distributions to total score of the common first-stage examination for university entrance II (in Japanese). Jpn. J. Appl. Statist., 10(1), 39-52.

Takeuchi, K. (1974). A test for multivariate normality. Behaviometrika, 1, 59-64.

(Received May 1998; Revised December 1998) 